\title{
THE GEOMETRY OF IMMERSIONS. I
}

\author{
BY \\ E. A. FELDMAN(1)
}

Introduction. For the past twenty-five years much of global differential geometry and topology has centered around what we now call differential topology. Much emphasis has been placed on those mathemathical ideas (vector bundles, their characteristic classes, and general position arguments) which depend only on the differentiable structure of manifolds and maps. In particular the work of Whitney and Thom (see Levine [1] and Whitney [1]-[6]) belong to that domain. Our principal aim in this paper is to show that the tools of differential topology can be suitably modified to become applicable to differential geometry. Our second goal is to use these tools to investigate some "higher-order" problems in differential geometry (that is, problems involving derivatives of order greater than 1).

We will assume that all manifolds discussed in this introduction are $C^{\infty}$ and satisfy the second axiom of countability. Furthermore we will use the words smooth, differentiable, and $C^{\infty}$ interchangeably.

The first problem encountered in such a program, is the one of finding the "natural" objects on which to build the theory. Of utmost importance is the fact that the theory of linear connections, central to differential geometry, must be built into these objects in a relatively meaningful way. The best objects that we found for this task were Pohl's (see Pohl [1]) pth order tangent bundles $T_{p}(X)$, where $X$ is a smooth manifold. The first section contains the definition of $T_{p}(X)$ and a résumé of their important properties. One of the important facts is the fact that the $T_{p}(X)$ satisfy the following short exact sequences of vector bundles:

$$
\left(E_{p}\right) 0 \rightarrow T_{p}(X) \rightarrow T_{p+1}(X) \rightarrow 0^{p+1} T_{1}(X) \rightarrow 0,
$$

where $0^{p}$ denotes the $p$-fold symmetric tensor product of vector bundles. Of equal importance is the fact that if $f: X \rightarrow Y$ is a smooth map there is induced a vector bundle homomorphism $T_{p}(f): T_{p}(X) \rightarrow T_{p}(Y)$ covering $f$.

Probably the most important aspect of this approach is that some connection theory can be built into it. It is a classical result (see Ambrose, Singer, and Palais [1]) that splittings of the exact sequence $0 \rightarrow T_{1}(X) \rightarrow T_{2}(X) \rightarrow 0^{2} T_{1}(X) \rightarrow 0$ are in 1-1 correspondence with symmetric linear connections on $X$. It is not hard

Received by the editors November 12, 1963 and, in revised form, May 15, 1964.

(1) This research was supported by the following contracts NONR-266(57) and NSF 19022 at Columbia University and NSF GP-2425 at Princeton University. Part of this paper is contained in the author's doctoral dissertation at Columbia University. 
to define linear connections on $T_{p}(X)$ with some additional properties, called pth order dissections and then to prove that the $p$ th order dissections are in 1-1 correspondence with splittings of the sequence

$$
0 \rightarrow T_{p}(X) \rightarrow T_{p+1}(X) \rightarrow 0^{p+1} T_{1}(X) \rightarrow 0 .
$$

The second section is devoted to this theory.

Let $X$ and $Y$ be smooth manifolds of dimensions $n$ and $N$ respectively. Let $\left(D^{(k)}\right), k=1,2, \cdots$, be a sequence of $k$ th order dissections on $Y$. Let $C(X, Y)$ denote the set of smooth maps from $X$ to $Y$ with a suitably strong topology, strong enough to insure that the immersions of $X$ in $Y$ are open in $C(X, Y)$. For each $k$ let $D^{(k)}$ also denote the map from $T_{k+1}(Y)$ to $T_{k}(Y)$ which splits

$$
0 \rightarrow T_{k}(Y) \rightarrow T_{k+1}(Y) \rightarrow 0^{k+1} T_{1}(Y) \rightarrow 0,
$$

induced by the $k$ th order dissection $D^{(k)}$. We can then form the map $D_{p}=D^{(1)} \cdots D^{(p-1)}: T_{p}(Y) \rightarrow T_{1}(Y)$. Hence for each $f \in C(X, Y)$ we have a vector bundle homomorphism $D_{p} T_{p}(f): T_{p}(X) \rightarrow T_{1}(Y)$ covering $f$. The map $D_{p} T_{p}(f)$ is called the pth order osculating map of $f$ with respect to the dissections $\left(D^{(k)}\right), k=1,2, \cdots$, on $Y$. It is to the study of the maps $D_{p} T_{p}(f)$ and the bundle $T_{p}(X)$ that we intend to apply the concepts of differential topology.

Let us fix the above data. The first question anyone familiar with Whitney's work (see Whitney [1]) would ask is whether there exists an $f \in C(X, Y)$ such that $D_{p} T_{p}(f): T_{p}(X) \rightarrow T_{1}(Y)$ is of maximal rank on each fiber. If $Y=R^{N}$, if the splittings $D^{(k)}$ are given by the linear structure on $R^{N}$, and if $N$ is very much larger than the fiber dimension of $T_{p}(X)$ Pohl gives an answer in the affirmative (Pohl [1, Theorem 2.4]). However using more powerful techniques we achieve the following result. If $N \geqq$ fiber $\operatorname{dim}\left(T_{p}(X)\right)+n$ or if $N \leqq$ fiber $\operatorname{dim}\left(T_{p}(X)\right)-n$ then the set of $f \in C(X, Y)$ such that $D_{p} T_{p}(f)$ is of maximal rank on each fiber, is open and dense in $C(X, Y)$ (see $\S 6)$. Pohl also poses a special case of the following problem: Let $f_{0}$, and $f_{1} \in C(X, Y)$ such that $D_{p} T_{p}\left(f_{0}\right)$ and $D_{p} T_{p}\left(f_{1}\right)$ are of maximal rank on each fiber. Does there exist a smooth homotopy $f_{t}: X \rightarrow Y, t \in[0,1]$, such that $D_{p} T_{p}\left(f_{t}\right): T_{p}(X) \rightarrow T(Y)$ is of maximal rank on each fiber. If all the $f_{t}$ are immersions such a homotopy is called a pth order regular homotopy. Our techniques yield the following results. If $f_{0}$ and $f_{1}$ are homotopic immersions and if $N \geqq$ fiber $\operatorname{dim}\left(T_{p}(X)\right)+n+1$ then $f_{0}$ and $f_{1}$ are $p$ th order regularly homotopic; and if $f_{0}$ and $f_{1}$ are regularly homotopic immersions, and if $N \leqq$ fiber $\operatorname{dim}\left(T_{p}(X)\right)-n-1$ then $f_{0}$ and $f_{1}$ are $p$ th order regularly homotopic (see $\S 7$ ).

Rather than proving these results directly, we prove them as corollaries of a general transversality theorem. Probably the clearest way of proving such theorems is to use a very useful abstraction of Thom's "strong" transversality theorem due to R. Abraham (see Abraham [1]). Abraham points out the crucial properties needed for such theorems to be true and thereby eliminates much of the 
confusion surrounding such theorems. $\S \S 4$ and 5 are devoted to the statement and proof of the main transversality theorem and some of its easy corollaries. $\S \S 8$ and 9 are devoted to proving some easy (and in some cases quite trivial) corollaries of the main theorems of $\S \S 6$ and 7 in order to give us a better geometric grasp of the maps $D_{p} T_{p}(f)$ and the bundles $T_{p}(X)$. A brief discussion of the characteristic classes and classifying maps of the $T_{p}(X)$ is included in $\S 8$. Finally the paper concludes with some counter-examples which indicate that the theorems of $\$ 6$ are optimal. In particular these examples tell us that the "higher-order counterpart" of Whitney's $(2 n-1)$ immersion theorem (Theorem 6 of Whitney [6]) is false.

The second part of this paper will apply this machinery, in particular the transversality theorem, to the study of some "Riemannian singularities." In particular the parabolic submanifold and the umbilic points of immersed hypersurfaces in $R^{N}$ will be discussed.

A more complete outline of this paper has appeared as a research announcement in the September, 1963 edition of the Bulletin of the American Mathematical Society. Since then we have changed the expression " $p$ th order symmetric linear connection" of the research announcement to the words " $p$ th order dissection" used above.

I would like to give special thanks to my advisor, Professor James Eells, for his patience, continued interest and many helpful suggestions. I would like to thank William Pohl for writing Pohl [1], as the ideas in his thesis greatly enriched my own and provided a foundation for much of the material in this thesis. Finally, I would like to thank I. Porteous for his interest and especially for bringing the work of Pohl to my attention.

\section{0 . Notations and conventions.}

(a) All manifolds discussed in this paper will be finite dimensional and all the connected components of a given manifold will have the same dimension. The manifolds will satisfy the second axiom of countability and will be of class $C^{\infty}$ We will use the adjectives smooth or differentiable to connote a manifold, or a map between manifolds of class $C^{\infty}$.

(b) If $X$ and $Y$ are smooth manifolds we will denote by $C^{k}(X, Y)$ the set of smooth maps from $X$ to $Y$ with the "fine" $C^{k}$-topology, and $C(X, Y)$ the set of smooth maps with the fine $C^{\infty}$-topology. This topology is merely the union of all the open sets of the $C^{k}$-topologies. It is a topology because it coincides with the inverse limit topology on $C(X, Y)$ viewing the $C^{k}(X, Y)$ with the obvious inclusion maps as an inverse mapping system. For further details on this topology we refer the reader to the appendix at the end of this paper. This appendix includes a proof of the fact that $C(X, Y)$ is a Baire space in the fine topology. All the theorems in this paper are stated in their " $C$ " ${ }^{\infty}$ " form; it is clear that they are also true " $C$ " " for $k$ sufficiently large. The $k$ can be determined by counting the 
number of derivatives used, and comparing that number with the restrictions imposed by the Sard theorem.

(c) If $\xi=\{\pi: E \rightarrow X\}$ is a smooth vector bundle we will denote by $S(\xi)$ the space of smooth sections of $\xi$ topologized as a subset of $C(X, E)$. We will denote by $F(X)$ the space of smooth real-valued functions $C(X, R)$. We will also denote the total space of the tangent vector bundle over a smooth manifold $X$ by $T_{1}(X)$ or $T(X)$.

(d) If $\xi=(\pi: E \rightarrow X)$ is a smooth vector bundle, we will mean by a linear (affine) connection on $\xi$ a covariant derivative, i.e., a map

such that

$$
D: S\left(T_{1}(X)\right) \times S(E) \rightarrow S(E)
$$

$$
\begin{aligned}
& D_{f x+g y} e=f D_{x} e+g D_{y} e \quad \begin{array}{l}
\text { for every } e \in S(E), f, g \in F(X) \\
\text { and } x, y \in S\left(T_{1}(X)\right) .
\end{array} \\
& D_{x} f e=f D_{x} e+x(f) e \begin{array}{l}
\text { for every } x \in S\left(T_{1}(X)\right), e \in S(E), \\
f \in F(X) \text { and } x(f) \text { is evaluation of } \\
\text { the vector field } x \text { on the function } f .
\end{array}
\end{aligned}
$$

(e) If $X$ is a smooth manifold denote by $F$ the sheaf of germs of smooth functions on $X$. If $U \subset X$ let $\Gamma\left(U,{ }^{*}\right)$ represent the section functor for sheaves. Let $F(U)=\Gamma(U, F)$. If $\xi=(\pi: E \rightarrow X)$ is a smooth vector bundle over $X$, let $E$ be the sheaf of germs of smooth cross-sections of $\xi$. $E$ is a sheaf of $F$-modules. It is clear that $\Gamma(U, E)$ is an $F(U)$-module and that $\Gamma(X, E)=S(E)$ and is an $F(X)$ module. For a full account of this "dialogue" between smooth vector bundles and their sheaves of germs of smooth sections we refer the reader to Koszul's notes (Koszul [1, p. 81]).

(f) Let $\xi=(\pi: E \rightarrow X)$ and $\eta=\left(\pi^{\prime}: E^{\prime} \rightarrow Y\right)$ be smooth vector bundles. Denote by $\operatorname{Hom}(\xi, \eta)$ (or by an abuse of notation $\operatorname{Hom}\left(E, E^{\prime}\right)$ ) the vector bundle over $X \times Y$ whose fiber over the point $(x, y) \in X \times Y$ is the vector space $\operatorname{Hom}\left(E_{x}, E_{y}^{\prime}\right)$. This set is made into a vector bundle in the obvious way. Let $D: X \rightarrow X \times X$ be the diagonal map, and let $\xi=(\pi: E \rightarrow X)$ and $\eta=\left(\pi^{\prime}: E^{\prime} \rightarrow X\right)$ be smooth vector bundles. We shall denote by $\operatorname{Hom}\left(E, E^{\prime}\right) \mid X$ the bundle $D^{-1}\left(\operatorname{Hom}\left(E, E^{\prime}\right)\right)$ where $D^{-1}($ ) means the "pull back" of the bundle over $X \times X$, to a bundle over $X$. This latter bundle (the one we denoted by $\left.\operatorname{Hom}\left(E, E^{\prime}\right) \mid X\right)$ is the bundle more usually denoted by $\operatorname{Hom}\left(E, E^{\prime}\right)$.

(g) The following number appears very frequently in our work, so we have set aside a special notation for it. Let

$$
v(n, p)=n+\frac{n(n+1)}{2}+\cdots+\frac{n(n+1)(n+2)(\cdots)(n+p-1)}{p !} .
$$

(h) Let $\xi=(\pi: E \rightarrow X)$ be a smooth vector bundle. Then we denote by $0^{p} E$ the $p$-fold symmetric tensor product of $E$ with itself. Let $\eta=\left(\pi^{\prime}: E^{\prime} \rightarrow Y\right)$ be 
another vector bundle, we connote by the expression " $g: E \rightarrow E$ ' is a vector bundle homomorphism covering $f: X \rightarrow Y$," the commutative diagram of smooth maps where $g$ maps each fiber $E_{x}$ linearly into the fiber $E_{f(x)}^{\prime}$ :

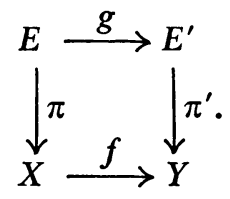

(i) Let $X$ be a smooth manifold. Let $N$ be a submanifold of $X$, and let $i: N \rightarrow X$ be the inclusion map. We say that $N$ is a regular submanifold if $i$ is a diffeomorphism into.

(j) Let $X$ be a smooth manifold, and let $\mathfrak{A}=\left(\phi_{i}, U_{i}\right), i=1,2, \cdots$, be a countable atlas for $X$. We say that $\mathfrak{A}$ is an admissible atlas if

1. $\phi_{i}\left(U_{i}\right) \cong R^{n}$

2. the open unit balls $B_{i}\left(B_{i}=\phi_{i}^{-1}\right.$ (unit ball in $\left.R^{n}\right)$ ) cover $X$;

3. the covering $\left(U_{i}\right), i=1,2, \cdots$, is locally finite.

1. $p$ th order tangent bundles. In this section we will review some facts about the $p$ th order tangent bundles. For a more complete discussion we refer the reader to Pohl [1].

Definition 1.1. Let $X$ be a smooth manifold of dimension $n$. Let $U$ be a coordinate neighborhood of $X$ with coordinate functions $\left(x_{1}, \cdots, x_{n}\right)$. Let $x \in U$, and let $\partial^{k} /\left.\partial x_{i_{1}} \cdots \partial x_{i_{k}}\right|_{x}$ represent the linear functional which sends $f \in F(U)$ into $\partial^{k} f /\left.\partial x_{i_{1}} \cdots \partial x_{i_{k}}\right|_{x}$. Let us fix $p \geqq 1$. Let $T_{p}(X)_{x}$ be the real vector space spanned by the linear functionals $\left\{\partial^{k} /\left.\partial x_{i_{1}} \cdots \partial x_{i_{k}}\right|_{x}, 1 \leqq k \leqq p, 1 \leqq i_{1} \leqq \cdots \leqq i_{k} \leqq n\right\}$. These functionals are clearly linearly independent. Let $T_{p}(X)=\bigcup_{x \in X} T_{p}(X)_{x}$. Let $V$ be a coordinate neighborhood of $x$ with coordinate functions $\left(y_{1}, \cdots, y_{n}\right)$ such that $x \in U \cap V$. We can give $T_{p}(X)$ the structure of a smooth vector bundle, if we relate the functionals $\left\{\partial^{k} /\left.\partial x_{i_{1}} \cdots \partial x_{i_{k}}\right|_{x}, 1 \leqq k \leqq p, 1 \leqq i_{1} \leqq \cdots \leqq i_{k} \leqq n\right\}$ to functionals $\left\{\partial^{s} /\left.\partial y_{j_{1}} \cdots \partial y_{j_{s}}\right|_{x}, 1 \leqq s \leqq p, 1 \leqq j_{1} \leqq \cdots \leqq j_{s} \leqq n\right\}$ by the obvious formula given by the chain rule for partial derivatives. $T_{p}(X)$ is called the $p t h$ order tangent bundle of $X$. The fiber dimension of $T_{p}(X)$ is $v(n, p)$.

REMARKS. Let $X$ be a smooth manifold of dimension $n$.

(a) Let $J_{0}^{[p]}(X)_{x}$ denote the $p$-jets of smooth functions of $X$ into $R$, which take the point $x \in X$ into the point $0 \in R$. Let $J_{0}^{[p]}(X)=\bigcup_{x \in X} J_{0}^{[p]}(X)_{x}$ be the corresponding bundle of $p$-jets. It is not hard to show that $T_{p}(X) \cong J_{0}^{[p]}(X)^{*}$.

(b) Let $U$ be a coordinate neighborhood of $X$, with coordinate functions $\left(x_{1}, \cdots, x_{n}\right)$. Then the $F(U)$-module $\Gamma\left(U, \boldsymbol{T}_{p}(X)\right)$ is free with the partial differential operators $\left\{\partial^{k} / \partial x_{i_{1}} \cdots \partial x_{i_{k}}, 1 \leqq k \leqq p, 1 \leqq i_{1} \leqq \cdots \leqq i_{k} \leqq n\right\}$ as a basis.

Definition 1.2. Let $X$ and $Y$ be smooth manifolds. Let $f \in C(X, Y)$. Then $f$ induces a map $f^{*}: F(Y) \rightarrow F(X)$ by function composition on the right by $f$. Let 
$T \in T_{p}(X)_{x}$. We can then define $T_{p}(f)_{x} T=T \circ f^{*}: F(Y) \rightarrow R$. It is an immediate consequence of the chain rule that $T_{p}(f)_{x} T \in T_{p}(Y)_{f(x)}$. Hence the $T_{p}(f)_{x}, x \in X$ defines a smooth homomorphism of vector bundles $T_{p}(f)$ which maps $T_{p}(X)$ into $T_{p}(Y)$ and covers the map $f: X \rightarrow Y$. The map $T_{p}(f)$ is called the pth differential of $f$.

Let $X$ be a smooth manifold and let $p \geqq 2$. It is clear that $T_{p-1}(X)$ is a vector subbundle of $T_{p}(X)$. Hence we have a well-defined cokernel $T_{p}(X) / T_{p-1}(X)$ and an epimorphism of vector bundles $\pi_{p-1}: T_{p}(X) \rightarrow T_{p}(X) / T_{p-1}(X)$. We now define a map $m_{p-1}: 0^{p} T_{1}(X) \rightarrow T_{p}(X) / T_{p-1}(X)$ as follows. Let $U$ be a coordinate neighborhood of $X$ with coordinates $\left(x_{1}, \cdots, x_{n}\right)$. Let $m_{p-1}\left(\partial /\left.\partial x_{i_{1}}\right|_{x} \circ \cdots \circ \partial /\left.\partial x_{i p}\right|_{x}\right)$ $=\pi_{p-1}\left(\partial^{p} /\left.\partial x_{i_{1}} \cdots \partial x_{i p}\right|_{x}\right)$. It is easy to see that $m_{p-1}$ is an isomorphism of vector bundles (see Pohl [1, p. 174]). We will throughout the the remainder of this paper denote the epimorphism $m_{p-1}^{-1} \pi_{p-1}: T_{p}(X) \rightarrow 0^{p} T_{1}(X)$ by $P_{p-1}$. We will collect the elementary properties of these bundles and maps in the following proposition.

Proposition 1.3. Let $X, Y$ and $Z$ be smooth manifolds, let $f \in C(X, Y)$ and let $g \in C(Y, Z)$.

(a) We have the following short exact sequences of vector bundles, for $p \geqq 2$ :

$$
0 \longrightarrow T_{p-1}(X) \underset{I_{p-1}}{\longrightarrow} T_{p}(X) \underset{P_{p-1}}{\longrightarrow} 0^{p} T_{1}(X) \longrightarrow 0
$$

where $I_{p-1}$ is the canonical inclusion map.

(b) $T_{p}(g f)=T_{p}(g) T_{p}(f)$, and $T_{p}\left(\mathrm{id}_{x}\right)=\mathrm{id}_{T_{p}}(X)$.

(c) The following diagram of vector bundles has exact rows and commutes:

$$
\begin{array}{r}
0 \longrightarrow T_{p-1}(X) \stackrel{I_{p-1}}{\longrightarrow} T_{p}(X) \stackrel{P_{p-1}}{\longrightarrow} 0^{p} T_{1}(X) \longrightarrow 0 \\
\qquad T_{p-1}(f) \quad T_{p}(f) \quad 0^{p} T_{1}(f) \\
0 \longrightarrow T_{p-1}(Y) \stackrel{I_{p-1}}{\longrightarrow} T_{p}(Y) \stackrel{P_{p-1}}{\longrightarrow} 0^{p} T_{1}(Y) \longrightarrow 0 .
\end{array}
$$

The proofs of this result are quite easy and can be found in Pohl [1].

Definition 1.4. Let $X$ be a smooth manifold. Let $\chi_{p}=\Gamma\left(X, T_{p}(X)\right)=S\left(T_{p}(X)\right)$. The elements of $\chi_{p}$ are called the pth order vector fields of $X$.

REMarks. Let $X$ be a smooth manifold. Let $\chi_{p}$ denote the $p$ th order vector fields on $X$.

(a) If we apply to a smooth vector bundle the functor which assigns to it its sheaf of germs of smooth sections and if we then apply to the sheaf of germs of smooth sections the global section functor, we get the following exact sequence of $F(X)$ modules:

$$
0 \longrightarrow \chi_{p-1} \stackrel{I_{p-1}}{\longrightarrow} \chi_{p} \stackrel{P_{p-1}}{\longrightarrow} 0_{F(x)}^{p} \chi_{1} \longrightarrow 0 .
$$

(b) If $x \in \chi_{1}$ and if $L \in \chi_{p}$ then we can define an element $\chi L$ in $\chi_{p+1}$ by function composition in $\operatorname{End}_{R} F(X)$. 
(c) The map $I_{p-1}$ is just the usual inclusion. The map $P_{p-1}$ has the following form. Let $\pi_{p-1}: \chi_{p} \rightarrow \chi_{p} / \chi_{p-1}$ be the usual coset map. Now define $m_{p-1}: 0_{F(x)}^{p} \chi_{1} \rightarrow \chi_{p} / \chi_{p-1}$ by

$$
m_{p-1}\left(x_{1} \circ \cdots \circ x_{p}\right)=\pi_{p-1}\left(x_{1} x_{2} \cdots x_{p}\right), \quad x_{i} \in \chi_{1} .
$$

Then $P_{p-1}=m_{p-1}^{-1} \pi_{p-1}$. This is a consequence of the manner in which the germs of sections functor and the global section functor act on vector bundle and sheaf of module homomorphisms respectively.

2. Dissections. Throughout this section fix $X$ as a smooth manifold.

Definition 2.1. A pth order dissection $(p>1)$ on $X$ is a linear connection $D$ on $T_{p}(X)$ satisfying the following two conditions.

(1) If $L \in \chi_{p-1}$, and if $x \in \chi_{1}$ then

$$
D_{x} L=x L .
$$

(2) Let $R$ be the curvature tensor of the connection $D$, i.e.,

$$
R: \chi_{1} \wedge_{F(x)} \chi_{1} \rightarrow \operatorname{Hom}_{F(x)}\left(\chi_{p}, \chi_{p}\right) .
$$

Then for every $x$ and $y \in \chi_{1}, R(x, y) \mid \chi_{p-1}$ is zero.

In the case $p=1$, a first order dissection will be a linear connection on $T_{1}(X)$ with torsion zero.

THEOREM 2.2. Let $X$ be a smooth manifold. pth order dissections of $X$ are in 1-1 correspondence with splittings of the short exact sequence of vector bundles

$$
0 \longrightarrow T_{p}(X) \stackrel{I_{p}}{\longrightarrow} T_{p+1}(X) \stackrel{P_{p}}{\longrightarrow} 0^{p+1} T_{1}(X) \longrightarrow 0 .
$$

Proof. It is easy to see that splittings of the above sequence are in 1-1 correspondence with splittings of the sheaves of $F$-modules

$$
0 \longrightarrow T_{p}(X) \stackrel{I_{p}}{\longrightarrow} T_{p+1}(X) \stackrel{P_{p}}{\longrightarrow} 0^{p+1} T_{1}(X) \longrightarrow 0 .
$$

Splittings of this exact sequence of sheaves are in turn in 1-1 correspondence with splittings of the short exact sequence of $F(X)$-modules

$$
0 \longrightarrow \chi_{p} \stackrel{I_{p}}{\longrightarrow} \chi_{p+1} \stackrel{P_{p}}{\longrightarrow} 0^{p+1} \chi_{1} \longrightarrow 0 .
$$

Let $x_{1}, \cdots, x_{p+1} \in \chi_{1}$, and let $D$ be a $p$ th order dissection defined on $X$. We want to define $\phi: 0^{p+1} \chi_{1} \rightarrow \chi_{p+1}$ as follows. Let

$$
\phi\left(x_{1} \circ \cdots \circ x_{p+1}\right)=x_{1} \cdots x_{p+1}-D_{x_{1}} x_{2} \cdots x_{p+1} .
$$

Pick $f \in F(X)$, then we immediately see that

$$
\phi\left(f x_{1} \circ \cdots \circ x_{p+1}\right)=f \phi\left(x_{1} \circ \cdots \circ x_{p+1}\right) .
$$


Now

$$
\begin{aligned}
\phi\left(x_{1}\right. & \left.\circ x_{2} \circ x_{3} \circ \cdots \circ x_{p+1}\right)-\phi\left(x_{2} \circ x_{1} \circ \cdots \circ x_{p+1}\right) \\
& =\left[x_{1}, x_{2}\right] \cdot x_{3} \cdots x_{p+1}-D_{x_{1}} x_{2} \cdots x_{p+1}+D_{x_{2}} x_{1} x_{3} \cdots x_{p+1} \\
& =\left[x_{1}, x_{2}\right] \cdot x_{3} \cdots x_{p+1}-D_{x_{1}} D_{x_{2}} x_{3} \cdots x_{p+1}+D_{x_{2}} D_{x_{1}} x_{3} \cdots x_{p+1} \\
& =\left(D_{\left[x_{1}, x_{2}\right]}-D_{x_{1}} D_{x_{2}}+D_{x_{2}} D_{x_{1}}\right)\left(x_{3} \cdots x_{p+1}\right)=0 .
\end{aligned}
$$

Let $i>1$. Then

$$
\begin{aligned}
& \phi\left(x_{1} \circ \cdots \circ x_{i} \circ x_{i+1} \circ \cdots \circ x_{p+1}\right)-\phi\left(x_{1} \circ \cdots \circ x_{i-1} \circ x_{i+1} \circ x_{i} \circ \cdots \circ x_{p+1}\right) \\
& \quad=x_{1} \circ \cdots \circ x_{i-1} \cdot\left[x_{i}, x_{i+1}\right] \cdot x_{i+2} \cdots x_{p+1}-D_{x_{1}} x_{2} \cdots x_{i-1}\left[x_{i}, x_{i+1}\right] \cdot x_{i+2} \cdots x_{p+1} \\
& \quad=x_{1} \cdots x_{i-1} \cdot\left[x_{i}, x_{i+1}\right] \cdot x_{i+2} \cdots x_{p+1}-x_{1} \cdot x_{2} \cdot x_{i-1} \cdot\left[x_{i}, x_{i+1}\right] \cdot x_{i+2} \cdots x_{p+1} \\
& \quad=0 .
\end{aligned}
$$

Hence $\phi$ is a well-defined map from $0^{p+1} \chi_{1}$ into $\chi_{p+1}$. Finally $P_{p} \cdot \phi\left(x_{1} \circ \cdots \circ x_{p+1}\right)$ $=x_{1} \circ \cdots \circ x_{p+1}$ by the definition of $P_{p}$. Hence $\phi$ is a splitting.

Conversely, let $\phi$ be a splitting of sequence $\left(^{*}\right)$. Let $f \in F(X), x \in \chi_{1}$ and $T \in \chi_{p}$. We define $D_{x} T=x \cdot T-\phi P_{p}(x \cdot T)$. We first notice that $P_{p} D_{x} T=0$ hence $D_{x} T \in \chi_{p}$. It is easy to see that $D_{f x} T=f D_{x} T$ and $D_{x} f T=x(f) T+f D_{x} T$. Therefore $D$ is a linear connection on $T_{p}(X)$. The definition immediately implies that $D_{x} T=x \cdot T$ if $T \in \chi_{p-1}$. Finally let $T \in \chi_{p-1}$, and $y \in \chi_{1}$. Then

$$
\begin{aligned}
& D_{x} D_{y} T-D_{y} D_{x} T-D_{[x, y]} T \\
& \quad=x y T-\phi P_{p}(x y T)-y x T+\phi P_{p}(y x T)-x y T+y x T \\
& \quad=\phi P_{p}(y x T-x y T)=\phi P_{p}([y, x] T)=0 .
\end{aligned}
$$

Therefore $D$ is a $p$ th order dissection.

Let $\phi$ be a given splitting, and let $\phi^{\prime}$ be splitting induced from the dissection $D$ induced by $\phi$. Now

$$
\begin{aligned}
\phi^{\prime}\left(x_{1} \circ \cdots \circ x_{p+1}\right) & =x_{1} \cdots x_{p+1}-D_{x_{1}} x_{2} \cdots x_{p+1} \\
& =x_{1} \cdots x_{p+1}-x_{1} \cdot x_{2} \cdots x_{p+1}-\phi P_{p}\left(x_{1} \cdots \cdots x_{p+1}\right) \\
& =\phi\left(x_{1} \circ \cdots \circ x_{p+1}\right)
\end{aligned}
$$

by the definition of $P_{p}$. Conversely let $D$ be a given $p$ th order dissection, and let $D^{\prime}$ be the dissection induced from the splitting $\phi$ induced by $D$. Let $x, y_{1}, \cdots, y_{p} \in \chi_{1}$. Then

$$
\begin{aligned}
D_{x}^{\prime} y_{1} \cdots y_{p} & =x \cdot y_{1} \cdots y_{p}-\phi\left(x_{1} \circ y \circ \cdots \circ y_{p}\right) \\
& =x \cdot y_{1} \cdots y_{p}-x \cdot y_{1} \cdots y_{p}+D_{x} y_{1} \cdots y_{p}=D_{x} y_{1} \cdots y_{p} .
\end{aligned}
$$


We know that $\chi_{p}$ is generated by linear combinations with coefficients in $F(X)$ of elements of the form $y_{1} \cdots y_{k}, 1 \leqq k \leqq p$ and $y_{i} \in \chi_{1}$. Due to the fact that both $D$ and $D^{\prime}$ are $p$ th order dissections the above relationship implies that $D^{\prime}=D$. This completes our proof.

THEOREM 2.3. Let $D$ and $\bar{D}$ be respectively $a p-1$ and a first order dissection on $X$. Then $D$ and $\bar{D}$ induce a pth order dissection on $X$.

Proof. Let $\phi: 0^{p} \chi_{1} \rightarrow \chi_{p}$ be the splitting of the sequence $\left(^{*}\right)$ of the last proof, induced by $D$. We will now define a map $\psi: \chi_{1} \otimes 0^{p} \chi_{1} \rightarrow \chi_{p+1}$ as follows. Let $x_{1}, \cdots, x_{p+1} \in \chi_{1}$, then

$$
\begin{aligned}
\psi\left(x_{1} \otimes\left(x_{2} \circ \cdots \circ x_{p+1}\right)\right)= & x_{1} \cdot \phi\left(x_{2} \circ \cdots \circ x_{p+1}\right) \\
& -\sum_{i=1}^{p+1} \phi\left(x_{2} \circ \cdots \circ \bar{D}_{x_{1}} x_{i} \circ x_{i+1} \circ \cdots \circ x_{p+1}\right) .
\end{aligned}
$$

It is an easy check to see that $\psi$ is a well-defined $F(X)$ linear map. We will now symmetrize $\psi$. Set

$$
\Phi\left(x_{1} \circ \cdots \circ x_{p+1}\right)=\sum_{i=1}^{p+1} \frac{1}{p+1} \psi\left(x_{i} \otimes\left(x_{1} \circ \cdots \circ \hat{x}_{i} \circ \cdots \circ x_{p+1}\right)\right)
$$

where $\wedge$ means that the term is omitted. $\bar{\phi}$ is clearly an $F(X)$-module homomorphism from $0^{p+1} \chi_{1}$ into $\chi_{p+1}$. That $\Phi$ splits the exact sequence of the last theorem follows from the definition of $\bar{\phi}$ and the definition of $\phi$.

Corollary 2.4. A given first order dissection on $X$ (a symmetric linear connection) induces $a$ pth order dissection on $X$ for all $p$.

REMARK. This last theorem and corollary were also discovered independently by Pohl (see Pohl [2]) and P. Libermann (Libermann [1]). Theorem 2.2 in the case $p=1$ is the well-known result of Ambrose, Singer, and Palais.

3. $p$ th order osculating maps and Abraham's transversality theorem. Theorem 2.2 permits us to represent $p$ th order dissections and the unique splitttings of $0 \rightarrow T_{p}(X) \rightarrow T_{p+1}(X) \rightarrow 0^{p+1} T_{1}(X) \rightarrow 0$ which the dissections induce by the same letter. We will also use that to represent the unique map from $T_{p+1}(X)$ into $T_{p}(X)$ which splits the above exact sequence on the left.

Definition 3.1. Let $X$ and $Y$ be smooth manifolds. Let $\left(D^{(k)}\right), k=1,2, \cdots$, be a sequence of $k$ th order dissections on $Y$ and let $f \in C(X, Y)$. Denote by $D_{p}$ the vector bundle homomorphism over $Y, D^{(1)} D^{(2)} \ldots D^{(p-1)}: T_{p}(Y) \rightarrow T_{1}(Y)$. The pth order osculating map of $f$ with respect to the dissections $\left(D^{(k)}\right)$ is the map $D_{p} T_{p}(f)$. It is a vector bundle homomorphism from $T_{p}(X)$ into $T_{1}(Y)$ covering $f$. Let $x \in X$. The pth order osculating space of $f$ at $x$ with respect to the $\left(D^{(k)}\right)$ is the vector subspace $D_{p} T_{p}(f)_{x}\left(T_{p}(X)_{x}\right)$ of $T_{1}(Y)_{(x)}$. Whenever no con- 
fusion can arise we will drop our explicit reference to the dissections $\left(D^{(k)}\right)$.

REMARK. Let $X$ and $Y$ be smooth manifolds. Let $D$ be a symmetric linear connection on $Y$. Let $\left(D^{(k)}\right)$ be the sequence of $k$ th order dissections on $Y$ that $D$ induces by Theorem 2.3. Let $f \in C(X, Y)$ and let $D_{p} T_{p}(f)$ be the $p$ th order osculating map of $f$. Let $x \in X$. Let $\Gamma_{x}=(c \in C(R, X) \mid c(0)=x)$. Let $c \in \Gamma_{x}$. Let $u_{t}(c)$ be the tangent vector to $c$ at $c(t)$. Let $v_{1}(c)_{t}=T_{1}(f) u_{t}(c)$. Let $v_{k}(c)_{t}$ be defined inductively by $D_{v_{1}(c) t} v_{k-1}(c)_{t} . v_{1}(c)_{t}, \cdots, v_{p}(c)_{t}$ are all elements of $T_{1}(Y)_{f(c(t))}$. Let $0 S_{p}(x)=$ the subspace of $T_{1}(Y)_{f(x)}$ spanned by the vectors $v_{1}(c)_{0}, \cdots, v_{p}(c)_{0}$, where $c$ runs through the set $\left.\Gamma_{x}\right\}$. Pohl shows (in Pohl $[2, \mathrm{p} .18]$ ) that $0 S_{p}(x)$ $=D_{p} T_{p}(f)\left(T_{p}(X)_{x}\right)$, which is the $p$ th order osculating space of $f$ at $x$.

REMARK. Let $Y=R^{N}$. Let $D$ be the symmetric linear connection given by the usual Riemannian structure on $R^{N}$. Let $\left(D^{(k)}\right)$ be the sequence of $k$ th order dissections on $Y$ induced by $D$. Let $\left(y_{1}, \cdots, y_{N}\right)$ be the usual global coordinate functions on $R^{N}$. Then an arbitrary $k$ th order vector field on $R^{N}$ is given by the formula

Then

$$
L=\sum_{1 \leqq i_{1} \leqq \ldots \leqq i_{s} \leqq N ; 1 \leqq s \leqq k} a_{i_{1} \ldots i_{s}}\left(y_{1}, \cdots, y_{N}\right) \frac{\partial^{s}}{\partial y_{i_{1}} \cdots \partial y_{i_{s}}}, \quad a_{i_{1} \ldots i_{\mathrm{s}}} \in F\left(R^{N}\right) .
$$

$$
D^{k-1} L=\sum_{1 \leqq i_{1} \leqq \ldots \leqq i_{s} \leqq N ; 1 \leqq s \leqq k-1} a_{i_{1} \ldots i_{s}}\left(y_{1}, \cdots, y_{N}\right) \frac{\partial^{s}}{\partial y_{i_{1}} \cdots \partial y_{t_{s}}}
$$

That is, $D^{(k-1)}$ sends the highest order derivatives (those of order $=k$ ) into zero. These two remarks may serve as a bridge between Definition 3.1 and more classical definitions with which the reader may be familiar.

Before proceeding any further we will finish this section with some definitions and statements concerning transversality of maps, including a statement of Abraham's transversality theorem.

Definition 3.2. Let $X$ and $Y$ be smooth manifolds and let $N$ be a smooth submanifold of $Y$. Let $f: X \rightarrow Y$ be a smooth map. We say that $f \in C(X, Y)$ is transverse to $N$ at $x \in X$ if either:

(1) $f(x) \notin N$ or

(2) $T_{1}(N)_{f(x)}+T_{1}(f)_{x}\left(T_{1}(X)_{x}\right)=T_{1}(Y)_{f(x)}$,

where $T_{1}(N)_{f(x)}$ is the tangent space to $N$ at $f(x)$. We say that $f$ is transverse to $N$ if it is transverse to $N$ at each $x \in X$. We will denote this situation by $f(X) \AA N$.

The following proposition is a trivial consequence of the definitions.

Proposition 3.3. Let $K$ be a submanifold of a smooth manifold $Z$ and let $Y$ be another smooth manifold. Then $K \times Y$ is a submanifold of $Z \times Y$. Let $\pi: Z \times Y \rightarrow Z$ be the projection map.

Let $f: X \rightarrow Z \times Y$ be a smooth map of a smooth manifold $X$ into $Z \times Y$, then $\pi f(X) \pitchfork K$ if and only if $f(X) \pitchfork K \times Y$.

Definition 3.4. Let $Y$ be a smooth manifold. A submanifold collection in $Y$ is a finite union $S=S_{1} \cup \cdots \cup S_{m}$ of disjoint regular smooth submanifolds $S_{i}$ such that 
(i) $\operatorname{dim} S_{k}>\operatorname{dim} S_{k+1}$, and

(ii) $S_{k} \cup S_{k+1} \cup \cdots \cup S_{m}$ is closed in $Y$.

The points of $S_{1}$ are called regular points. The dimension (codimension) of a submanifold collection will be the dimension (codimension) of its regular points.

Let $X$ be another smooth manifold. A smooth map $f: X \rightarrow Y$ is said to be transverse to $S$ at $x \in X$, if it is transverse to the submanifold $S_{k}$ containing $f(x)$, or $f(x) \notin S$. Finally if $f: X \rightarrow Y$ is transverse to $S$ at each point $x \in X$ (denoted $f(X) \pitchfork S)$, then $f^{-1}(S)$ is a submanifold collection of $X$,

$$
f^{-1}(S)=f^{-1}\left(S_{i}\right) \cup \cdots \cup f^{-1}\left(S_{m}\right)
$$

describes a decomposition of $S$ satisfying conditions (i) and (ii) above, and $\operatorname{codim} S_{\imath}=\operatorname{codim} f^{-1}\left(S_{i}\right)$ for $i=1,2, \cdots, m$.

Definition 3.5. Let $S=S_{1} \cup \cdots \cup S_{m}$ be a submanifold collection of $R^{N}$. Let $\operatorname{dim} S_{i}=s_{i}$. Let $G_{N, k}$ denote the Grassmann manifold of $k$-planes through the origin of Euclidean $N$-space. Let $G_{i}: S_{i} \rightarrow G_{N, s_{i}}$ be the Gauss tangential map on $S_{i}$. That is, $G_{i}(x)$ is the parallel translate of the $s_{i}$-plane through $x$ given by $T_{1}\left(S_{i}\right)_{x}$ through the origin. $G_{i}$ is a smooth map for each $i$. Let $y \in S_{k}, k>1$. Let $\left(x_{i}\right)$ be a sequence of points of $S_{j}, j<k$ which converges to $y$. Let us look at the set $\left\{G_{j}\left(x_{i}\right)\right\}=\left\{p_{i}\right\} .\left\{p_{i}\right\}$ is an infinite sequence of points in $G_{N, s_{j}}$, and hence it must have a cluster point. Let $C\left(\left\{p_{i}\right\}\right)$ be the set of such cluster points. Let $p \in C\left(\left\{p_{i}\right\}\right)$. We would like $p$ to contain $G_{k}(y)$ as a hyperplane through the origin. If this is the case for all arbitrary sequences of points $\left\{x_{i}\right\}$ contained in $S_{j}$, and converging to $y$, and for all $S_{j}, j<k$, we say the submanifold collection $S$ is cohesive at $y$. If this is true for all $y \in S_{2} \cup \cdots \cup S_{m}$, then we say that the submanifold collection $S$ is cohesive.

Let $S=S_{1} \cup \cdots \cup S_{l}$ be a submanifold collection of $Y$. Let $y \in S_{k}$ for $k>1$. Then we say that $S$ is cohesive at $y$ if there exists a coordinate neighborhood $(U, \phi)$ of $y$, such that $\phi(U \cap N)$ is cohesive at $\phi(y)$. If $S$ is cohesive at all $y \in S_{2} \cup \cdots \cup S_{l}$, then we say that $S$ is cohesive.

REMARK. If $M$ is a closed submanifold with boundary of a smooth manifold $Y$, then it is easy to see that $M$ is a cohesive submanifold collection of $Y$, as $M=\operatorname{Int}(M) \cup \operatorname{Bd}(M)$. We have described the property of being cohesive in terms of a given "decomposition" of a point set $S$, as a manifold collection $S=S_{1} \cup \cdots S_{k}$. It is possible that a given point set $S$ have two decompositions into submanifold collections, one of which is cohesive, and another one which isn't. For example consider the point set $S$ consisting of the $x y$-plane and the $z$-axis in $R^{3}$. Then $S$ has the following decomposition: $S=(x y$-plane $-\{0\}) \cup z$-axis, and $S=(x y$-plane $-\{0\}) \cup(z$-axis $-\{0\}) \cup\{0\}$. The first decomposition is not cohesive, while the second one is. The following generalization of Thom's and Whitney's transversality theorems partially motivates our interest in cohesive submanifold collections. 
Proposition 3.6. Let $X$ and $Y$ be smooth manifolds. Let $S=S_{1} \cup \cdots \cup S_{m}$ be a submanifold collection in $Y$. Let

$$
B=\{f \in C(X, Y) \mid f(X) \pitchfork S\} .
$$

(a) If $\operatorname{codim} S>\operatorname{dim} X$, then $B$ is open and dense in $C(X, Y)$.

(b) If $S$ is a cohesive submanifold collection of $Y$, then $B$ is open and dense in $C(X, Y)$.

Proof. Let $N \subset Y$ be a regular submanifold of $Y$. Let $N_{c}$ be a compact subset of $Y$, which is contained in $N$, and let $K$ be a compact subset of $X$. Let $A_{K, N_{-}}=\left\{f \in C(X, Y) \mid f\right.$ is transverse to $N_{c}$ at each $\left.x \in K\right\}$. The standard techniques tell us that $A_{K, N_{c}}$ is open and dense in $C(X, Y)$. The Baire category theorem then yields the "dense" statements, of part (a) and (b), as well as the density of the set $B$, under the condition that $S$ be either a regular submanifold, or a finite disjoint family of regular submanifolds with no additional assumptions. The "open" statement of part (a) is a triviality. Hence the only thing at all new here is the "openness" statement of part (b).

Let us adopt the following notations. Let $S^{k}=S_{k} \cup S_{k+1} \cup \cdots \cup S_{m}$, let $\operatorname{dim} S_{j}=s_{j}$, let $G_{N, k}$ be the Grassmannian defined in 3.5, and let $N=\operatorname{dim} Y$. Let $\pi: J(X, Y) \rightarrow X \times Y$ be the fiber bundle of 1-jets of maps from $X$ to $Y$. As is well known, $\pi^{-1}(x \times y)$ is just the set of linear maps from $T_{1}(X)_{x}$ into $T_{1}(Y)_{y}$. Let $L$ be a linear subspace of $T_{1}(Y)_{y}$, we say that $\alpha \in J(X, Y)_{(x, y)}$ is transverse to $L$ if

$$
\alpha\left(T_{1}(X)_{x}\right)+L=T_{1}(Y)_{y} .
$$

Let $(x, y) \in X \times S$, then $y \in S_{k}$ for a unique $k$. Then there exists a closed subset $K_{x, y} \subset \pi^{-1}(x, y)$ such that $\alpha \in K_{x, y}$ if and only if $\alpha$ is not transverse to $T_{1}\left(S_{k}\right)_{y}$. Let $K=\bigcup_{(x, y) \in X \times s} K_{x, y}$. If we can show that $K$ is closed we have completed our proof.

Let $\left(k_{i}\right)$ be a sequence of points in $K$ converging to $k$. To show that $k \in K$, let $\pi\left(k_{i}\right)=\left(x_{i}, y_{i}\right)$ and let $\pi(k)=(x, y)$. We know $y_{i} \rightarrow y$ and that $x_{i} \rightarrow x$; furthermore $y \in S$ because $S$ is closed. If $y \in S_{1}$, we can pick a coordinate neighborhood $U$ of $y$ such that $U \cap S^{2}=\varnothing$, and $U \cap S_{1}$ is a hyperplane of dimension $s_{1}$. In this case it is easy to see that $k \in K_{x, y}$. The second case is when there exists but a finite number of distinct $y_{i}$ unequal to $y$. Then pick a coordinate neighborhood $U$ of $y$, which excludes all these $y_{i}$. Hence the $k_{i}$ of interest are all elements of $\bigcup_{\tilde{x} \in X} K_{\tilde{x}, y}$, which is locally isomorphic to $U \times K_{\tilde{x}, y}$ where $U$ is a coordinate neighborhood of $\tilde{x} \in X$. Hence this set is closed and therefore $k \in K_{x, y}$.

The case of interest is the one where there exists an infinite number of $y_{i}$, and $y \notin S_{1}$. Assume $y \in S_{k}, k>1$, hence there exists a coordinate neighborhood $U$ of $y$ such that $U \cap S^{k+1}=\varnothing$. Now the $\left\{y_{i}\right\}$ can be partitioned into sequences $\left\{y_{i_{1}}\right\}, \cdots,\left\{y_{i_{k}}\right\}$, such that $\left\{y_{i_{1}}\right\} \subset S_{1}, \cdots,\left\{y_{i_{k}}\right\} \subset S_{k}$, at least one of which is infinite. 
If the sequence $\left\{y_{i_{k}}\right\}$ is infinite (distinct too), then the corresponding $k_{i_{k}}$ can be shown to converge to $K$ in the same manner as in case 1 . So the last open case is the one where $\left\{y_{i_{j}}\right\}$ is an infinite number of distinct points of $S_{j}$ and $j \neq k$. We are interested in the subsequence $\pi^{-1}\left(x_{i_{j}}, y_{i_{j}}\right)=k_{i_{j}}$ which converges to $k$.

If $\alpha \in \pi^{-1}(x, y)$ is not transverse to a subspace of $T_{1}(Y)_{y}$ containing $T_{1}\left(S_{k}\right)_{y}$, then it is not transverse to $T_{1}\left(S_{k}\right)_{y}$. Let $U$ be a coordinate neighborhood of $y$. Let $G_{j}: S_{j} \cap U \rightarrow G_{N, s_{j}}$ be the associated Gauss map. Now let $p_{i_{j}}=G_{j}\left(y_{i_{j}}\right) \in G_{N, s_{j}}$. Let $V$ be a coordinate neighborhood of $x \in X$, and let

$$
\gamma: \pi^{-1}(U \times V) \rightarrow U \times V \times M(n, N)
$$

be the induced diffeomorphism where $n=\operatorname{dim} X$, and $M(n, N)$ is the set of $n \times N$ matrices. Let $\gamma\left(k_{i_{j}}\right)=\left(x_{i_{j}}, y_{i_{j}}, h_{i_{j}}\right)$ and let $\gamma(k)=(x, y, h)$. Let $p_{\alpha}$ be an accumulation point of $\left\{p_{i_{j}}\right\}$ in $G_{N, s_{j}}$. It is an easy consequence of the continuity of $\gamma$ and of $G_{j}$, that $h$ is not transverse to $p_{\alpha}$. However $p_{x}$ contains $G_{k}\left(T_{1}\left(S_{k}\right)_{y}\right)$ as a linear subspace, by the hypothesis that $S$ be cohesive. Hence $k \in K_{x, y}$, and we have demonstrated our theorem.

Let $X, Y$ and $Z$ be smooth manifolds. Let $S$ be a submanifold collection of $Z$. Let $A$ be a subset of $C(X, Y)$. Let $\lambda: A \rightarrow C(X, Z)$ be a continuous map. Let

$$
A_{s}=\{f \in A \mid \lambda g(X) \pitchfork S\} .
$$

If $S$ is either cohesive or, if $\operatorname{dim} X<\operatorname{codim} S$, then Proposition 3.6 coupled with the continuity of $\lambda$ tells us that $A_{s}$ is open in $A$. Let $E v_{\lambda}: A \times X \rightarrow Z$ be the continuous map defined by $E v_{\lambda}(f)(x) . E v_{\lambda}$ is called the $\lambda$-evaluation map.

Definition 3.7. Let $(x, f) \in X \times A$. $\lambda$ is called finitely evaluation transveral to $S$ at $(x, f)$ if there exists a neighborhood $W_{f}$ of $f$ as a point in $A$, a neighborhood $U$ of $x$, and a finite dimension cell $E \ni 0$, such that:

1. For every $g \in W_{f}$ there exists a continuous map $H_{g}: E \rightarrow A$ such that $H_{g}(0)=g$, and

2. $H_{g}$ enables us to define a map $D_{g}: E \times U \rightarrow Z$ by $D_{g}(\alpha, x)=\lambda H_{g}(\alpha)(x)$ with the properties:

(i) $D_{g}: E \times U \rightarrow Z$ is $C^{\infty}$,

(ii) $D_{g}(E \times U) \pitchfork S$.

The following theorem is an immediate corollary of the proof of Abraham's transversality theorem (see Abraham [1]).

THEOREM 3.8. Let $X, Y$ and $Z$ be smooth manifolds. Let $S \subset Z, A \subset C(X, Y)$, and let $\lambda: A \rightarrow C(X, Z)$ be continuous. If $A$ is a Baire space, if $\lambda$ is finitely evaluation transversal to $S$ at each point of $A \times X$, and if $S$ is either cohesive or, if $\operatorname{dim} X<\operatorname{codim} S$, then

$$
A_{S}=\{g \in A \mid \lambda g(X) \pitchfork S\}
$$

is open and dense in $A$. 
REMARK. In this paper we only use this result in the case where $\operatorname{dim} X<\operatorname{codim} S$. However in its sequels we use the cohesive result.

\section{A transversality theorem for osculating maps.}

Definition 4.1. Let $\xi=(\pi: E \rightarrow B)$ be a smooth fiber bundle with fiber $F$. The pair of spaces $\left(K, K^{\prime}\right)$ is said to be an admissible submanifold collection of $E$ if

1. $K=K_{1} \cup \cdots \cup K_{m}$ is a submanifold collection of $E$,

2. $K^{\prime}=K_{1}^{\prime} \cup \cdots \cup K_{m}^{\prime}$ is a submanifold collection of $F$,

3. there exists an atlas $\mathfrak{A}=\left\{\left(U, \phi_{U}\right)\right\}$ of $E$ such that $\phi_{U}\left(\pi^{-1}(U) \cap K_{i}\right)=U \times K_{i}^{\prime}$, $i=1,2, \cdots m$, for all $\left(U, \phi_{U}\right) \in \mathfrak{A}$.

REMARK. $K$ is cohesive if and only if $K^{\prime}$ is, and $\operatorname{codim} K=\operatorname{codim} K^{\prime}$.

Definition 4.2. Let $\xi=(\pi: E \rightarrow B)$ and $\xi^{\prime}=\left(\pi^{\prime}: E^{\prime} \rightarrow B^{\prime}\right)$ be vector bundles. Denote by $\operatorname{Hom}\left(E, E^{\prime}\right)$ the vector bundle over $B \times B^{\prime}$ with fiber $\operatorname{Hom}\left(E_{x}, E_{y}^{\prime}\right)$ over $(x, y) \in B \times B^{\prime}$ induced in the obvious way from $\xi$ and $\xi^{\prime}$.

We will now fix the following data throughout this section. Let $X$ and $Y$ be smooth manifolds of dimension $n$ and $N$ respectively. Let $\left(D^{(k)}\right), k=1,2, \cdots$, be a sequence of $k$ th order dissections on $Y$. Let $f \in C(X, Y)$. We can then define a map $D(f): X \rightarrow \operatorname{Hom}\left(T_{p}(X), T_{1}(Y)\right)$, which is a cross-section over the graph of $f$ defined by

$$
D(f)(x)=D_{p} T_{p}(f)_{x} \in \operatorname{Hom}\left(T_{p}(X)_{x}, T_{1}(Y)_{f(x)}\right) .
$$

Hence $D: C(X, Y) \rightarrow C\left(X, \operatorname{Hom}\left(T_{p}(X), T_{1}(Y)\right)\right)$ by associating to each $f \in C(X, Y)$ its $p$ th order osculating map.

THEOREM 4.3. a. The map $D$ defined above is continuous.

b. Let $\left(K, K^{\prime}\right)$ be an admissible submanifold collection of $\operatorname{Hom}\left(T_{p}(X), T_{1}(Y)\right)$. Then $D$ is finitely evaluation transveral to $K$ at each point of $C(X, Y) \times X$.

The Abraham theorem provides the following theorem as an immediate corollary of 4.3 , because $C(X, Y)$ is a Baire space.

THEOREM 4.4. Let $\left(K, K^{\prime}\right)$ be an admissible submanifold collection of $\operatorname{Hom}\left(T_{p}(X), T_{1}(Y)\right)$. Let

$$
A=\{f \in C(X, Y) \mid D(f)(X) \pitchfork K\} .
$$

If either $\operatorname{codim} K^{\prime}>\operatorname{dim} X$, or if $K^{\prime}$ is a cohesive submanifold collection, then $A$ is open and dense in $C(X, Y)$.

Proof of Theorem 4.3. Pick $f \in C(X, Y)$. Let $\mathfrak{A}=\left(U_{i}\right), \quad i=1,2, \cdots$, and $\mathfrak{B}=\left(V_{i}\right), i=1,2, \cdots$, be coordinate covers of $X$ and $Y$ such that $f\left(U_{\alpha}\right) \subset V_{\alpha}$ for $\alpha=1,2, \cdots$. We can also assume that $\left\{U_{\alpha} \times V_{\alpha}\right\}, \alpha=1,2, \cdots$, trivialize the bundle $\operatorname{Hom}\left(T_{p}(X), T_{1}(Y)\right)$ and furthermore are compatible with the admissible submanifold collection $\left(K, K^{\prime}\right)$. Let us restrict our attention to a single $U_{i}$ and $V$ 
which we will denote by $U$ and $V$, respectively. Let $\left(x_{1}, \cdots, x_{n}\right)$ be coordinate functions on $U$. Let $\left(y_{1}, \cdots, y_{N}\right)$ be coordinate functions on $V$, and let $\pi: \operatorname{Hom}\left(T_{p}(X), T_{1}(Y)\right) \rightarrow X \times Y$ be the projection map. Hence the functionals $\partial^{r}\left|\partial x_{i_{1}} \cdots \partial x_{i_{r}}\right|_{x}, 1 \leqq i_{1} \leqq \cdots \leqq i_{r} \leqq n ; 1 \leqq r \leqq p$, are a basis for $T_{p}(X)_{x}$, and the functionals $\partial /\left.\partial y_{j}\right|_{y}, 1 \leqq j \leqq N$, are a basis for $T_{1}(Y)_{y}$, where $(x, y) \in U \times V$. These will enable us to pick local coordinates for $\pi^{-1}(U \times V)$. We can define the functions $\left(\phi_{i}^{k}, \phi_{i_{1}, i_{2}}^{k}, \cdots, \phi_{i_{1}, i_{2}, \ldots, i_{s}}^{k}, 1 \leqq k \leqq N, 1 \leqq i_{1} \leqq \cdots \leqq i_{s} \leqq n, 1 \leqq s \leqq p\right)$ as coordinate functions in $\operatorname{Hom}\left(T_{p}(X)_{x}, T_{1}(Y)_{y}\right)$ as follows. Let

$$
a \in \operatorname{Hom}\left(T_{p}(X), T_{1}(Y)\right)_{(x, y)} .
$$

Then

$$
a\left(\left.\frac{\partial^{r}}{\partial x_{i_{1}} \cdots \partial x_{i_{r}}}\right|_{x}\right)=\left.\sum_{k=1}^{N} a_{i_{1} \ldots i_{r}}^{k} \frac{\partial}{\partial y_{k}}\right|_{y} .
$$

We then define $\phi_{i_{1} \ldots i_{r}}^{k}(a)=a_{i_{1} \ldots i_{r}}^{k}$. Hence the functions $\left(x_{i}, y_{j}, \phi_{i_{1} \ldots i_{r}}^{k}\right)$ are local coordinates for $\pi^{-1}(U \times V)$.

Let $D^{k}: \Gamma\left(V, \boldsymbol{T}_{k+1}(\boldsymbol{Y})\right) \rightarrow \Gamma\left(V, \boldsymbol{T}_{\boldsymbol{k}}(\boldsymbol{Y})\right)$ be the splitting induced by the $k$ th order dissection $D^{(k)}$. Then we define smooth functions ${ }^{(k)} \Gamma_{j_{1} \ldots j_{t}}^{l_{1} \ldots l_{s}}, 1 \leqq l_{1} \leqq \cdots \leqq l_{s} \leqq N$ $1 \leqq j_{1} \leqq \cdots \leqq j_{t} \leqq N ; 1 \leqq s \leqq k ; 1 \leqq t \leqq k+1$ by the formula

$$
D^{(k)} \frac{\partial^{t}}{\partial y_{j_{1}} \cdots \partial y_{j_{t}}}=\sum_{1 \leqq l_{1} \leqq \ldots \leqq l_{s} \leqq N ; 1 \leqq s \leqq k}{ }^{(k)} \Gamma_{j_{1} \ldots j_{t}}^{l_{1} \ldots l_{s}} \frac{\partial^{s}}{\partial y_{l_{1}} \cdots \partial y_{l_{s}}} .
$$

Let $f_{J}=y_{j} \cdot f$ and let $y=f(x)$. We know that

$$
\begin{aligned}
T_{p}(f)_{x}\left(\left.\frac{\partial^{r}}{\partial x_{i_{1}} \cdots \partial x_{i_{r}}}\right|_{x}\right)= & \left.\sum_{k=1}^{N} \frac{\partial^{r} f_{k}(x)}{\partial x_{i_{1}} \cdots \partial x_{i_{r}}} \frac{\partial}{\partial y_{k}}\right|_{y} \\
& +\left.\sum_{1 \leqq j_{1} \leqq \ldots \leqq j_{l} \leqq N ; 2 \leqq l \leqq r} C_{i_{1} \ldots i_{r}}^{j_{1} \ldots j_{l}} \frac{\partial^{l}}{\partial y_{j_{1}} \cdots \partial y_{j_{l}}}\right|_{y} .
\end{aligned}
$$

Each $C_{i_{1} \ldots i_{r}}^{j_{1} \ldots j_{1}}$ is a polynomial with integer coefficients in the partials of the $f_{j}$ 's with respect to the $x_{i}$ 's. Each monomial in $C_{i_{1} \ldots i_{r}}^{j_{1} \ldots j_{l}}$ contains a partial derivative of an $f_{j}$ of order strictly less than $r$. Finally we have

$$
D_{p} T_{p}(f)_{x}\left(\left.\frac{\partial^{r}}{\partial x_{i_{1}} \cdots \partial x_{i_{r}}}\right|_{x}\right)=\left.\sum_{k=1}^{N}\left(\frac{\partial^{r} f_{k}}{\partial x_{i_{1}} \cdots \partial x_{i_{r}}}+d_{i_{1} \ldots i_{r}}^{k}\right) \frac{\partial}{\partial y_{k}}\right|_{y} .
$$

Each $d_{i_{1} \ldots i_{r}}^{k}$ is a polynomial with integer coefficients in the $C_{i_{1}}^{j_{1} \ldots i_{r}}, s \leqq r$, and the functions $\Gamma$ defined above. Each monomial in $d_{i_{1} \ldots i_{r}}^{k}$ contains at least one of the functions $\Gamma$, and at least one partial derivative of order $<r$. Hence we have a formula of the form of $\left({ }^{* *}\right)$ above for each coordinate neighborhood $\left(U_{\alpha}\right)$ of $X$. Therefore it follows immediately from the definition of the fine topology on $C(X, Y)$ and $C\left(X, \operatorname{Hom}\left(T_{p}(X), T_{1}(Y)\right)\right)$ that the mapping $D$ is continuous. This completes the proof of part (a) of 4.3. 
It remains to show that $D$ is finitely evaluation transversal at $(x, f)$. For each $x \in X$, and $f \in C(X, Y)$. Let us keep the notation of the first part of the proof.

$N$. B. We shall assume that the coordinate functions $\left(x_{1}, \cdots, x_{n}\right)$ on $U$ are $x$-centered. We shall also "identify" $U$ and $V$ respectively with $R^{n}$ and $R^{N}$ without making explicit reference to the coordinate diffeomorphisms. This last convention although an abuse of notation will shorten already lengthy formulas.

Finally we will start calling $U$ by the name of $U^{\prime}$, as we want to reserve the letter $U$ to be the neighborhood of $x$ which corresponds to the same lettered neighborhood in Definition 3.4.

Let $B$ be the open unit ball in $U^{\prime}$. Let $\tilde{V}$ be the open 2-ball in $U^{\prime}$. Then we have $B \subset \bar{B} \subset \tilde{V} \subset \tilde{V}-\subset U^{\prime}$. Let $W_{f^{\prime}}=\{g \in C(X, Y) \mid g(U) \subset V\} . W_{f^{\prime}}$ is an open neighborhood of $f$. Let $g \in W_{f^{\prime}}$. Then $g \mid U^{\prime}: R^{n} \rightarrow R^{N}$ by our abuse of notation. Let $E^{\prime}=R^{v(n, p) \cdot N}$. Let an element of $E^{\prime}$ be given by a $v(n, p) \cdot N$-tuple of elements $\left(\alpha_{j_{1} \ldots j_{k}}^{i} ; 1 \leqq i \leqq N ; 1 \leqq j_{1} \leqq \cdots \leqq j_{k} \leqq n, 1 \leqq k \leqq p\right)$. Let

$$
y_{i}\left(G\left(\alpha, x^{\prime}\right)\right)=g_{i}\left(x^{\prime}\right)+\sum_{j=1}^{n} \alpha_{j}^{l} x_{j}^{\prime}+\cdots+\sum_{1 \leqq j_{1} \leqq \ldots \leqq j_{p} \leqq n} \alpha_{j_{1} \ldots j_{p}}^{i} x_{j_{1}}^{\prime} \cdots x_{j_{p}}^{\prime} .
$$

Hence this formula defines a smooth map

$$
G: E^{\prime} \times R^{n} \rightarrow R^{N}
$$

Let $\Phi \in F(X)$ such that $0 \leqq \Phi \leqq 1$ and

(i) $\Phi \mid \vec{B}=1$,

(ii) $\Phi \mid X-\tilde{V}=0$.

Now the map $G$ above can be interpreted as a map from $E^{\prime} \times U^{\prime} \rightarrow V$. We now define

$$
g\left(\alpha, x^{\prime}\right)= \begin{cases}\phi G\left(\alpha, x^{\prime}\right)+(1-\phi) G\left(0, x^{\prime}\right), & x^{\prime} \in U^{\prime}, \\ g\left(x^{\prime}\right), & x^{\prime} \in X-\tilde{V} .\end{cases}
$$

[The addition takes place in $V$ which is identified with $R^{N}$.] $g\left(\alpha, x^{\prime}\right): E^{\prime} \times X \rightarrow Y$ is clearly a well-defined smooth map, furthermore $g\left(0, x^{\prime}\right)=g\left(x^{\prime}\right)$. Therefore we have defined a map

$$
\tilde{H}: W_{f^{\prime}} \times E^{\prime} \rightarrow C(X, Y)
$$

given by $\tilde{H}(g, \alpha)\left(x^{\prime}\right)=g\left(\alpha, x^{\prime}\right)$, and $\tilde{H}$ is clearly continuous. We can furthermore define a continuous map

$$
H_{g}^{\prime}: E^{\prime} \rightarrow C(X, Y)
$$

for each $g \in W_{f^{\prime}}$ by $H_{g}^{\prime}(\alpha)=\tilde{H}(g, \alpha)$. $H_{g}^{\prime}$ restricted to a suitable neighborhood of $0 \in E^{\prime}$, will be the $H_{g}$ of Definition 3.4.

We can now define a continuous map

$$
\widetilde{D}: E^{\prime} \times W_{f^{\prime}} \times B \rightarrow \pi^{-1}\left(U^{\prime} \times V\right)
$$


by $\tilde{D}\left(\alpha, g, x^{\prime}\right)=D \tilde{H}(g, \alpha)\left(x^{\prime}\right)$. $\tilde{D}$ is clearly continuous, furthermore if we fix $g$ we form $D_{g}^{\prime}: E^{\prime} \times B \rightarrow \pi^{-1}\left(U^{\prime} \times V\right)$ by $D_{g}^{\prime}\left(\alpha, x^{\prime}\right)=D H_{g}^{\prime}(\alpha)\left(x^{\prime}\right)$, and we find that $D_{g}^{\prime}$ is smooth.

We recall that our neighborhoods $U^{\prime} \times V$ are compatible with the bundle structure of $\operatorname{Hom}\left(T_{p}(X), T_{1}(Y)\right)$ as well as the admissible submanifold collection $\left(K, K^{\prime}\right)$. For simplicity we will denote the fiber of $\operatorname{Hom}\left(T_{p}(X), T_{1}(Y)\right)$ by $F$. Hence we have a diffeomorphism

$$
\rho: \pi^{-1}\left(U^{\prime} \times V\right) \rightarrow U^{\prime} \times V \times F .
$$

Furthermore $\rho\left(\pi^{-1}\left(U^{\prime} \times V\right) \cap K\right)=U^{\prime} \times V \times K^{\prime}$. Finally let $q: U^{\prime} \times V \times F \rightarrow F$ be the obvious projection. These enable us to define the continuous map

$$
q \rho \tilde{D}: E^{\prime} \times W_{f^{\prime}} \times B \rightarrow F .
$$

$q \rho \tilde{D}$ is a smooth map for each fixed $g \in W_{f^{\prime}}$. Let us fix $\left(g, x^{\prime}\right) \in W_{f^{\prime}} \times B$. Then $q \rho \tilde{D}$ defines a smooth map from $E^{\prime} \rightarrow F$. We recall that $\operatorname{dim} E^{\prime}=\operatorname{dim} F=v(n, p) \cdot N$. Hence it is possible to talk about the jacobian determinant of this map $q \rho \widetilde{D}$ (for fixed $\left.\left(g, x^{\prime}\right)\right): E^{\prime} \rightarrow F$. Let $J\left(\alpha, g, x^{\prime}\right): E^{\prime} \times W_{f^{\prime}} \times B \rightarrow \boldsymbol{R}$ be that jacobian determinant of $q \rho \tilde{D}$ for each fixed $\left(g, x^{\prime}\right)$. It is an immediate consequence of our constructions that $J$ is a continuous map.

LEMma 4.5. $J(0, f, x) \neq 0$.

Before proving this lemma we will show that the lemma implies that $D$ is finitely evaluation transversal. By the continuity of $J$ there exists a neighborhood $E$ of $0 \in E^{\prime}$, such that $E$ is a cell, a neighborhood $W_{f}$ of $f, W_{f} \subset W_{f^{\prime}}$, and a neighborhood $U$ of $x, U \subseteq B$, such that

$$
J: E \times W_{f} \times U \rightarrow \text { reals, and } J(\alpha, g, x) \neq 0
$$

for any $(\alpha, g, x) \in E \times W_{f} \times U$. This statement implies that for each $g \in W_{f}$, the smooth map

$$
\rho \tilde{D}_{g}: E \times U \rightarrow F
$$

has maximal rank at each point $(\alpha, x)$. Let $D_{g}=\widetilde{D}_{g} \mid E \times U$. Then we therefore know that for each $g \in W_{f}, q \rho D_{g}(E \times U) \pitchfork K^{\prime}$. However this implies that

$$
\rho D_{g}(E \times U) \pitchfork U \times V \times K^{\prime}
$$

by Proposition 3.3. Hence $D_{g}(E \times U) \pitchfork K \cap\left(\pi^{-1}(U \times V)\right)$ for each $g \in W_{f}$. This proves that $D$ is finitely evaluation transversal.

Proof of Lemma 4.5. We recall that $U^{\prime}$ and $V$ are coordinate neighborhoods of $X$ and $Y$ respectively with coordinate function $\left(x_{1}, \cdots, x_{n}\right)$ and $\left(y_{1}, \cdots, y_{N}\right)$, respectively. We shall furthermore assume that $U^{\prime}$ is $x$-centered (i.e., $x=(0, \cdots, 0)$ in $R^{n}$ ). Let us look at the map $H_{f}^{\prime}(\alpha)$ on $B$, the unit ball in $U^{\prime} . H_{f}^{\prime}(\alpha): B \rightarrow V$. It then follows from our definitions that 


$$
\frac{\partial^{s} y_{i}\left(H_{f}^{\prime}(\alpha)\right)}{\partial x_{r_{1}} \cdots \partial x_{r_{s}}}=\frac{\partial^{s} y_{i}(f)}{\partial x_{r_{1}} \cdots \partial x_{r_{s}}}+K \alpha_{r_{1} \ldots r_{s}}^{i}+\xi_{r_{1} \ldots r_{s}}^{i}, \quad K>0 \text { real, }
$$

where $\xi_{r_{1} \ldots r_{s}}^{i}$ is a polynomial in the $\alpha_{j_{1} \ldots j_{t}}^{i}$ and the $x_{j}$ 's, with integer coefficients. Furthermore each monomial contains at least one such $x_{j}$. Hence $\xi_{r_{1} \ldots r_{s}}^{i}$ is $o(1)$ (i.e., $\xi_{r_{1} \ldots r_{s}}^{i} \rightarrow 0$ as $x \rightarrow 0$ ). Furthermore this formula implies

$$
\begin{aligned}
\frac{\partial \xi_{r_{1} \ldots r_{s}}^{i}}{\partial \alpha_{j_{1} \ldots j_{t}}^{i}} & =0, \quad k \neq i, \\
\left.\frac{\partial \xi_{r_{1} \ldots r_{s}}^{i}}{\partial \alpha_{j_{1} \ldots j_{t}}^{i}}\right|_{x=0} & = \begin{cases}\left(\delta_{j_{1}, r_{1}} \cdots \delta_{r_{s}, j_{s}}\right) K & \text { if } s=t \\
0 & \text { if } s \neq t\end{cases}
\end{aligned}
$$

Hence by formula $\left({ }^{* *}\right)$ at the beginning of the proof,

$$
\phi_{r_{1} \ldots r_{s}}^{k}\left(q \rho D_{f}^{\prime}\left(\alpha, x^{\prime}\right)\right)=\frac{\partial^{s} y_{k} f}{\partial x_{r_{1}} \cdots \partial x_{r_{s}}}+K \alpha_{r_{1} \ldots r_{s}}^{k}+\xi_{r_{1} \ldots r_{s}}^{k}+d_{r_{1} \ldots r_{s}}^{k}(\alpha),
$$

$d_{r_{1} \ldots r_{s}}^{k}(\alpha)$ is a polynomial in integer coefficients in the $\Gamma$ 's, $\alpha$ 's and partials of the $f_{i}$ 's with respect to the $x_{j}$ 's. Furthermore each $d_{r_{1} \ldots r_{s}}^{k}(\alpha)$ is a sum of expressions of the form $\delta_{1} \cdots \delta_{a} \gamma_{1} \cdots \gamma_{b}, a \geqq 1$ and $b \geqq 1$, where $\gamma_{i}$ is one of the functions $\Gamma$, defined by the dissections, and each $\delta_{i}$ is an expression of the form

$$
\left(\frac{\partial^{t} y_{i}\left(H_{f}^{\prime}(\alpha)\right)}{\partial x_{k_{1}} \cdots \partial x_{k_{t}}}+\alpha_{k_{1} \ldots k_{t}}^{i}+\xi_{k_{1} \ldots k_{t}}^{i}\right), \quad t<s
$$

We will now calculate $\partial d_{r_{1} \ldots r_{s}}^{k}(\alpha) /\left.\partial \alpha_{r_{1}^{\prime} \ldots r_{s}^{\prime}}^{k^{\prime}}\right|_{x=0}$ for $s^{\prime} \geqq s$. By the above formula we know that

$$
\frac{\partial \delta_{i}}{\partial \alpha_{r_{1}^{\prime} \ldots r_{s}^{\prime}}^{k^{\prime}}}=\frac{\partial \xi_{l_{1} \ldots l_{t}}^{i}}{\partial \alpha_{r_{1}^{\prime} \ldots r_{s}^{\prime}}^{k^{\prime}}}
$$

which is $o(1)$ and

$$
\frac{\partial \gamma_{j}}{\partial \alpha_{r_{1}^{\prime} \ldots r_{s}^{\prime}}^{k^{\prime}}}=\sum_{k=1}^{N} \frac{\partial \gamma_{j}}{\partial y_{k}} \frac{\partial y_{k}\left(H_{f}^{\prime}(\alpha)\right)}{\partial \alpha_{r_{1}^{\prime} \ldots r r_{s}^{\prime}}^{k^{\prime}}}
$$

which is $o(1)$. Hence $\partial d_{r_{1} \ldots r_{s}}^{k}(\alpha) / \partial \alpha_{r_{1}^{\prime} \ldots r_{s}^{\prime}}^{k^{\prime}}$ for $s^{\prime} \geqq s$ is $o(1)$ for each $\alpha \in E^{\prime}$. But recall that the $U$-coordinates are $x$-centered. Hence

$$
\left.\frac{\partial \phi_{r_{1} \ldots r_{s}}^{k}\left(q \rho \hat{D}_{f}\left(\alpha, x^{\prime}\right)\right)}{\partial \alpha_{r_{1}^{\prime} \ldots r_{s}^{\prime}}^{k^{\prime}}}\right|_{(\alpha, 0)}= \begin{cases}0 & \text { if } s^{\prime}>s, \\ K \delta_{k, k^{\prime}} \delta_{r_{1}, r_{1}^{\prime}} \ldots \delta_{r_{s}, r_{s}}(K>0) & \text { if } s^{\prime}=s .\end{cases}
$$

Hence the determinant of the above horrendous matrix is $\neq 0$ and equal to $J(\alpha, f, x)$ therefore $J(0, f, x) \neq 0$.

This completes the proof of the lemma and theorem. 
5. Two easy generalizations of the transversality theorems. Let $X$ and $Y$ be smooth manifolds of dimension $n$ and $N$, respectively. Let $\left(D^{(k)}\right), k=1,2, \cdots$, be a sequence of $k$ th order dissections on $Y$. Let $E$ be a vector sub-bundle of $T_{p}(X)$ over $X$, of fiber dimension $e$. Let $f \in C(X, Y)$. Then we have the $p$ th order osculating map $D_{p} T_{p}(f): T_{p}(X) \rightarrow T_{1}(Y)$. This induces a map

$$
D_{E}: C(X, Y) \rightarrow C\left(X, \operatorname{Hom}\left(E, T_{1}(Y)\right)\right)
$$

defined by $D_{E}(f)(x)=D_{p} T_{p}(f)_{x} \mid E_{x}$, for each $f \in C(X, Y)$ and each $x \in X$. Hence if we fix an $f \in C(X, Y), D_{E}(f): X \rightarrow \operatorname{Hom}\left(E, T_{1}(Y)\right)$ induces a cross-section of Hom $\left(E, T_{1}(Y)\right)$ over the graph of $f$ in $X \times Y$.

Let $\pi_{E}: \operatorname{Hom}\left(E, T_{1}(Y)\right) \rightarrow X \times Y$ be the bundle projection. Let $\left(K, K^{\prime}\right)$ be an admissible submanifold collection of $\operatorname{Hom}\left(E, T_{1}(Y)\right)$ of co-dimension $k$.

THEOREM 5.1. Let $X$ and $Y$ be smooth manifolds of dimension $n$ and $N$, respectively. Let $\left(D^{(k)}\right), k=1,2 \cdots$, be a sequence of $k$ th order dissections on $Y$. Let $E$ be a vector sub-bundle of $T_{p}(X)$, of fiber dimension e. Let $\left(K, K^{\prime}\right)$ be an admissible submanifold collection of $\operatorname{Hom}\left(E, T_{1}(Y)\right)$. If $K^{\prime}$ is either cohesive or if $\operatorname{codim} K^{\prime}>n=\operatorname{dim} X$, then the set of $f \in C(X, Y)$ such that $D_{E}(f)(X) \pitchfork K$ is open and dense in $C(X, Y)$.

Proof. $E$ is a vector sub-bundle of $T_{p}(X)$. Let $F=T_{p}(X) / E$. The fiber of $F$ is $R^{v(n, p)-e}$. In that $X$ satisfies the second axiom of countability,

$$
\operatorname{Hom}\left(T_{p}(X), T_{1}(Y)\right) \cong \operatorname{Hom}\left(E, T_{1}(Y)\right) \oplus \operatorname{Hom}\left(F, T_{1}(Y)\right),
$$

where $\oplus$ is the Whitney sum. Let $\pi, \pi_{E}$ and $\pi_{F}$ be the bundle projection of $\operatorname{Hom}\left(T_{p}(X), T_{1}(Y)\right), \operatorname{Hom}\left(E, T_{1}(Y)\right)$, and $\operatorname{Hom}\left(F, T_{1}(Y)\right)$, respectively. Let $U \times V$ be a mutual, trivializing coordinate neighborhood for each of these bundles. Hence

$$
\begin{aligned}
& \pi_{E}^{-1}(U \times V) \cong U \times V \times \operatorname{Hom}\left(R^{e}, R^{N}\right), \\
& \pi_{F}^{-1}(U \times V) \cong U \times V \times \operatorname{Hom}\left(R^{v(n, p)-e}, R^{N}\right), \text { and } \\
& \pi^{-1}(U \times V) \cong U \times V \times \operatorname{Hom}\left(R^{v(n, p)}, R^{N}\right) .
\end{aligned}
$$

$\operatorname{Hom}\left(R^{e}, R^{N}\right) \oplus \operatorname{Hom}\left(R^{v(n, p)-e}, R^{N}\right) \rightarrow \tilde{\tilde{\phi}} \operatorname{Hom}\left(R^{v(n, p)}, R^{N}\right)$, where $\phi$ is the isomorphism induced by the ismorphism between $\operatorname{Hom}\left(T_{p}(X), T_{1}(Y)\right)$ and $\operatorname{Hom}\left(E, T_{1}(Y)\right) \oplus \operatorname{Hom}\left(F, T_{1}(Y)\right)$. Hence $\operatorname{Hom}\left(R^{v(n, p)}, R^{N}\right) \leftrightarrow \widetilde{\tilde{\phi}} \operatorname{Hom}\left(R^{e}, R^{N}\right) \times$ $\operatorname{Hom}\left(R^{v(n, p)-e}, R^{N}\right)$ as manifolds. $K=K_{1} \cup \cdots \cup K_{m}$ is an admissible submanifold collection of $\operatorname{Hom}\left(E, T_{1}(Y)\right)$ of codimension $k$. Hence we can assume that the atlases were so chosen that $\pi_{E}^{-1}(U \times V) \cap K_{i}=U \times V \times K_{i}^{\prime}$, where $K^{\prime}=K_{1}^{\prime} \cup \cdots \cup K_{m}^{\prime}$. Let $\tilde{K}_{i}^{\prime}=\phi\left(K_{i}^{\prime} \times \operatorname{Hom}\left(R^{v(n, p)-e}, R^{N}\right)\right)$. Then $\tilde{K}^{\prime}=\tilde{K}_{1}^{\prime} \cup \cdots \cup \tilde{K}_{m}^{\prime}$ is a submanifold collection of $\operatorname{Hom}\left(R^{N}\right)$ of codimension $k$. Furthermore $\tilde{K}^{\prime}$ is cohesive if $K^{\prime}$ is. Let $\widetilde{K}=\widetilde{K}_{1} \cup \cdots \cup \widetilde{K}_{m}$ be the submanifold collection of $\operatorname{Hom}\left(T_{p}(X), T_{1}(Y)\right)$ given by $\tilde{K}_{i} \cap \pi^{-1}(U \times V)=U \times V \times \tilde{K}_{i}^{\prime}$, where $U \times V$ 
is picked as above. Then $\left(\tilde{K}, \tilde{K}^{\prime}\right)$ is an admissible submanifold collection of $\operatorname{Hom}\left(T_{p}(X), T_{1}(Y)\right)$ of codimension $k$, which is cohesive if $\left(K, K^{\prime}\right)$ is. Finally we must show that $D_{p}(f)(X) \AA \tilde{K}$ implies that $D_{E}(f)(X) \AA K$.

Since transversality is a local property, we will check it locally. Let $f \in C(X, Y)$. Let $x \in X$. Let $U \times V$ be a locally trivializing neighborhood of $\operatorname{Hom}\left(E, T_{1}(Y)\right)$, $\operatorname{Hom}\left(F, T_{1}(Y)\right)$, and $\operatorname{Hom}\left(T_{p}(X), T_{1}(Y)\right)$, compatible with the admissible submanifold collections $\left(K, K^{\prime}\right)$ and $\left(\widetilde{K}, \widetilde{K}^{\prime}\right)$, such that $x \in U$ and $f(x) \in V$. Let

$$
\theta_{E}: \pi_{E}^{-1}(U \times V) \rightarrow \operatorname{Hom}\left(R^{e}, R^{N}\right)
$$

and

$$
\theta: \pi^{-1}(U \times V) \rightarrow \operatorname{Hom}\left(R^{v(n, p)}, R^{N}\right)
$$

be the local projection onto the fiber. Let $\pi_{1}: \operatorname{Hom}\left(R^{e}, R^{N}\right) \times \operatorname{Hom}\left(R^{v(n, p)-e}, R^{N}\right)$ $\rightarrow \operatorname{Hom}\left(R^{e}, R^{N}\right)$ be the usual projection. Then let $\lambda: \operatorname{Hom}\left(R^{v(n, p)}, R^{N}\right) \rightarrow \operatorname{Hom}\left(R^{e}, R^{N}\right)$ be given by $\pi_{1} \phi^{-1}$. We know that $\lambda\left(\tilde{K}^{\prime}\right)=\left(K^{\prime}\right)$ and $\lambda \theta=\theta_{E}$. Assume $D_{p}(f)$ is transverse to $\widetilde{K}$ at $x$. Then Proposition 3.3 implies that $\theta D_{p}(f)$ is transverse to $\widetilde{K}^{\prime}$ at $x$. Therefore we have

$$
T_{1}\left(\theta D_{p}(f)\right)_{x} T_{1}(X)_{x}+T_{1}\left(\tilde{K}^{\prime}\right)_{\theta D_{p}(f)(x)}=T_{1}\left(\operatorname{Hom}\left(R^{v(n, p)}, R^{N}\right)\right)_{\theta D_{p}(f)(x)} .
$$

Applying $T_{1}(\lambda)$ to both sides yields

$$
T_{1}\left(\theta_{E} D_{E}(f)\right)_{x} T_{1}(X)_{x}+T_{1}\left(K^{\prime}\right)_{\theta_{E} D_{E}(f)(x)}=T_{1}\left(\operatorname{Hom}\left(R^{e}, R^{N}\right)\right)_{\theta_{E} D_{E}(f)(x)} .
$$

H nce $\theta_{E} D_{E}(f)$ is transverse to $K^{\prime}$ at $x$, which implies that $D_{E}(f)$ is transverse to $K$ at $x$ by Proposition 3.3. Hence $D_{p}(f)(X) \pitchfork K$ implies $D_{E}(f)(X) \pitchfork K$. Now apply Theorem 4.4 . with $\tilde{K}$ as the admissible submanifold collection.

THEOREM 5.2 (EXTENSION THEOREM). Let $X$ and $Y$ be smooth manifolds. Let $\left(D^{(k)}\right), k=1,2, \cdots$, be a sequence of kth order dissections on $Y$. Let $A$ be a closed subset of $X$, and let $E$ be a vector sub-bundle of $T_{p}(X)$. Let $\left(K, K^{\prime}\right)$ be an admissible submanifold collection of $\operatorname{Hom}\left(E, T_{1}(Y)\right)$. Let $f_{0} \in C(X, Y)$, such that $D_{E}\left(f_{0}\right)(A) \pitchfork K$. Let

$$
C_{A}\left(f_{0}\right)=\left\{g \in C(X, Y)|g| A=f_{0} \mid A\right\} .
$$

If either $K$ is a cohesive submanifold collection of $\operatorname{Hom}\left(E, T_{1}(Y)\right)$ or if $\operatorname{codim} K>\operatorname{dim} X$, then the set of $g \in C_{A}\left(f_{0}\right)$ such that $D_{E}(f) \pitchfork K$ is dense in $C_{A}\left(f_{0}\right)$.

Proof. Consider the smooth manifold $X-A$. Let $\delta$ be a positive function on $X-A$ such that $\delta \rightarrow 0$ as $x \rightarrow A$. Let $\left(d_{q}\right)$ be a set of complete metrics on $\left(\operatorname{Hom}\left(T_{q}(X), T_{q}(Y)\right)\right)$ for $q=1,2, \cdots$, compatible with the manifold topology. Then $d_{q}$ induces a metric $d_{q}^{\prime}$ on $\operatorname{Hom}\left(T_{q}(X-A), T_{q}(Y)\right)$ for each $q$. Pick $f \in C_{A}\left(f_{0}\right)$. Let $\mathfrak{A}=\left(U_{i}, \phi_{i}\right), i \in I$, be an admissible atlas for $X-A$. Then set 


$$
\left(S_{\delta}(f)\right)_{i}=\left\{g \in C(X-A, Y) \mid \sum_{q=0}^{\infty} \frac{1}{2^{q}} \frac{d_{q}^{\prime}\left(T_{q}(g)_{x}, T_{q}(f)_{x}\right)}{1+d_{q}^{\prime}\left(T_{q}(g)_{x}, T_{q}(f)_{x}\right)}<\delta(x) \text {, for } x \in \bar{B}_{i}\right\} .
$$

Set $S_{\delta}(f)=\bigcap_{i=1}^{\infty}\left(S_{\delta}(f)\right)_{i}$. $S_{\delta}(f)$ is an open neighborhood of $f \mid X-A$ in $C(X-A, Y)$. $S_{\delta}(f)$ defines a set $S_{\delta}(f)_{A} \subset C_{A}\left(f_{0}\right)$ as follows. If $h \in S_{\delta}(f)$ we define a smooth map $h^{\prime} \in C_{A}\left(f_{0}\right)$ by

$$
h^{\prime}= \begin{cases}h & \text { on } X-A, \\ f_{0} & \text { on } A\end{cases}
$$

Let $W \subset C_{A}\left(f_{0}\right)$ be a neighborhood of $f$ in the relative topology. Then there exists a positive continuous function $\delta_{W}$ on $X-A$ such that $\delta_{W}(x) \rightarrow 0$ as $x \rightarrow A$, with the property that $S_{\delta_{W}}(f)_{A} \subset W$. Now by Theorem 5.1 applied to $X-A$, there exists $g \in S_{\delta_{W}}(f)_{A}$ such that $D_{E}(g)(X-A) \pitchfork K$. If we let $g^{\prime}: X \rightarrow Y$ be the corresponding map in $S_{\delta_{W}}(f)_{A}$, then $D_{E}\left(g^{\prime}\right)(X) \AA K$ which proves our contention.

\section{6. $p$ th order embedding and immersion theorems.}

Definition 6.1. Let $X$ and $Y$ be smooth manifolds of dimension $n$ and $N$ respectively. Let $f \in C(X, Y)$. Let $\left(D^{(k)}\right), k=1,2, \cdots$, be a sequence of $k$ th order dissections of $Y$. Let $D_{p} T_{p}(f): T_{p}(X) \rightarrow T_{1}(Y)$ be the $p$ th order osculating map of $f$ with respect to the dissections. We say that $f$ is pth order nondegenerate at $x \in X$ (with respect to the given dissections) if $D_{p} T_{p}(f)_{x}: T_{p}(X)_{x} \rightarrow T_{1}(Y)_{f(x)}$ is of maximal rank. We say that $f$ is pth order nondegenerate (with respect to the dissections $\left.D^{(k)}\right)$ if $f$ is $p$ th order nondegenerate at every $x$ in $S$.

REMARK. $f$ is $p$ th order nondegenerate is equivalent to the bundle homomorphism $D_{p} T_{p}(f): T_{p}(X) \rightarrow T_{1}(Y)$ being injective or surjective on each fiber as $v(n, p) \leqq N$ or $v(n, p) \geqq N$, respectively. Moreover if $N \geqq v(n, p)$ and if $f$ is nondegenerate of order $p$, it is then nondegenerate of order $q$ for $q \leqq p$. Furthermore, nondegeneracy of order 1 (where dissections are not needed) means $f$ is an immersion. Hence if $N \geqq v(n, p)$ all $p$ th order nondegenerate maps are immersions.

THEOREM 6.2. Let $X$ and $Y$ be smooth manifolds of dimension $n$ and $N$, respectively. Let $\left(D^{(k)}\right), k=1,2, \cdots$, be a sequence of $k$ th order dissections on $Y$. Then if $N \leqq v(n, p)-n$, or if $N \geqq v(n, p)+n$ the set of pth order nondegenerate maps with respect to the $\left(D^{(k)}\right), k=1,2, \cdots$, are open and dense in $C(X, Y)$.

Proof. We will pick out a finite set of admissible submanifolds $K_{i}$ of $\operatorname{Hom}\left(T_{p}(X), T_{1}(Y)\right)$, with the property that $f$ is a $p$ th order nondegenerate map if and only if $D(f)(X) \cap K_{i}=\varnothing$ for all $i$, where

$$
D: C(X, Y) \rightarrow C\left(X, \operatorname{Hom}\left(T_{p}(X), T_{1}(Y)\right)\right)
$$

is the map defined at the beginning of $\S 4$. Furthermore $K=\bigcup_{i} K_{i}$ will clearly be a submanifold collection. See Levine [1, p. 6]. 
We first pick out the submanifolds $K_{i}^{\prime}$ of the fiber $\operatorname{Hom}\left(R^{v(n, p)}, R^{N}\right)$. The set $\operatorname{Hom}\left(R^{v(n, p)}, R^{N}\right)$ can be viewed as the $v(n, p)$ by $N$ matrices. Let $K_{i}^{\prime}$ be the submanifold which corresponds to the matrices of rank $i$. Let

$$
\pi: \operatorname{Hom}\left(T_{p}(X), T_{1}(Y)\right) \rightarrow X \times Y
$$

be the bundle projection. Let $U \times V$ be a locally trivializing coordinate neighborhood for the bundle $\operatorname{Hom}\left(T_{p}(X), T_{1}(Y)\right)$. Then we can define $K$ by the relation $\pi^{-1}(U \times V) \cap K_{i}=U \times V \times K_{i}^{\prime}$. Hence the $K_{i}, i=0,1,2, \cdots, \min (N, v(n, p))-1$ are a set of mutually disjoint admissible submanifolds of $\operatorname{Hom}\left(T_{p}(X), T_{1}(Y)\right)$. It is clear that $f$ is $p$ th order nondegenerate if and only if $D(f)(X) \cap K_{i}=\varnothing$, for all $i$.

We know that the codimension of $K_{i}^{\prime}=$ the codimension of $K_{i} . K_{i}^{\prime}$ is a submanifold of codimension equal to $v(n, p) N-i(N+v(n, p)-i)$. The $K_{i}^{\prime}$ of least codimension is $K_{v(n, p)-1}^{\prime}$ if $N \geqq v(n, p)$ or $K_{N-1}^{\prime}$ if $N \leqq v(n, p)$. If $n<$ codimension of $K_{v(n, p)-1}^{\prime}$ where $N \geqq v(n, p)$ or if $n<$ codimension $K_{N-1}^{\prime}$ where $N \leqq v(n, p)$, this theorem is implied by Theorem 4.4. The codimension of $K_{v(n, p)-1}^{\prime}$ is $N-v(n, p)+1$. Hence $n<N-v(n, p)+1$ if and only if $N \geqq v(n, p)+n$. Similarly the codimension of $K_{N-1}^{\prime}$ is $v(n, p)-N+1$. Hence $n<v(n, p)-N+1$ if and only if $N \leqq v(n, p)-n$.

Using the same hypotheses found in 6.2, we have the following corollaries.

COROLlaRY 6.3. If $N \geqq v(n, p)+n$, and if $X$ is compact, the pth order nondegenerate embeddings are open and dense in $C(X, Y), p>1$.

Proof. This is an immediate consequence of the Whitney embedding theorem (Whitney [1]) and Theorem 6.2 above.

REMARK. For $p=1,6.2$ says that if $N \geqq 2 n$, the immersions are open and dense in $C(X, Y)$ which is just the Whitney immersion theorem (Whitney [1]).

Let $I(X, Y)$ and $E(X, Y)$ be respectively the set of immersions and the set of embeddings in $C(X, Y)$ with the induced topology. It is well known that they are both open.

COROLlaRY 6.4. If $N \leqq v(n, p)-n$, then the set of pth order nondegenerate immersions (embeddings) is open and dense in $I(X, Y)(E(X, Y))$. If, furthermore $2 n \leqq N \leqq v(n, p)-n$ the set of pth order nondegenerate immersions is open and dense in $C(X, Y)$.

COROLlaRY 6.5. Let us assume the hypotheses of 6.2 Let us further assume that $A \subset X$ is a closed submanifold. Let $f_{0}: X \rightarrow Y$ be a map which is pth order nondegenerate on $A$. Let $C_{A}\left(f_{0}\right)=\left\{g \in C(X, Y)|g| A=f_{0} \mid A\right\}$. Then if $N \geqq v(n, p)+n$ or if $N \leqq v(n, p)-n$, the set of $g \in C_{A}\left(f_{0}\right)$ which are pth order nondegenerate on $X$ is dense in $C_{A}\left(f_{0}\right)$.

REMARK. By taking various permutations and combinations of these theorems 
and corollaries and those of Whitney and Thom many more corollaries could be stated. So we will stop now.

7. $p$ th order regular homotopies. Let $I=[0,1]$ be the closed unit interval. Let $X$ and $Y$ be smooth manifolds of dimension $n$ and $N$ respectively. Let $\left(D^{(k)}\right), k=1,2, \cdots$, be a sequence of $k$ th order dissections on $Y$. If $F: X \times I \rightarrow Y$ we will denote by $f_{t}$ the map $F \mid X \times\{t\}$.

Definition 7.1. Let $f_{0}$ and $f_{1}$ be $p$ th order nondegenerate immersions (embeddings) with respect to the $D^{(k)}$, of $X$ into $Y$. Then a smooth homotopy $F: X \times I \rightarrow Y$ between $f_{0}$ and $f_{1}$ is called a pth order regular homotopy (pth order isotopy) between $f_{0}$ and $f_{1}$, with respect to the $D^{(k)}$, if for each $t \in I, f_{t}: X \rightarrow Y$ is a $p$ th order nondegenerate immersion (embedding). If such a homotopy (isotopy) exists the two immersions (embeddings) are called pth order regularly homotopic (pth order isotopic) with respect to the $D^{(k)}$. If no confusion is present (and there usually is not) we will drop the appendage, "with respect to $\left(D^{(k)}\right), k=1,2, \cdots$."

REMARK. $p$ th order regular homotopies ( $p$ th order isotopies) are regular homotopies (isotopies).

REMARK. For the sake of not having to fuss about manifolds with boundary, we will assume that all homotopies are maps $F: X \times \tilde{I} \rightarrow Y$, where $\tilde{I}=(0-\varepsilon, 1+\varepsilon)$ and $\varepsilon$ is a small positive number and where $f_{t}=f_{1}, t \geqq 1$, and $f_{t}=f_{0}, t \leqq 0$.

THeOREM 7.2. Let $X$ and $Y$ be smooth manifolds of dimension $n$ and $N$, respectively. Let $\left(D^{(k)}\right), k=1,2, \cdots$, be a sequence of, $k$ th order dissections on $Y$. Let $f_{0}$ and $f_{1}$ be two pth order nondegenerate immersions.

(a) If $N \geqq v(n, p)+n+1$, and if $f_{0}$ and $f_{1}$ are homotopic then they are pth order regularly homotopic.

(b) If $N \geqq \operatorname{Max}(2 n+3, v(n, p)+n+1)$, if $X$ is compact, if $f_{0}$ and $f_{1}$ are pth order nondegenerate embeddings, and if they are homotopic, then $f_{0}$ and $f_{1}$ are pth order isotopic.

(c) If $n<N \leqq v(n, p)-n-1$, and if $f_{0}$ and $f_{1}$ are regularly homotopic, then they are pth order regularly homotopic.

(d) If $n<N \leqq v(n, p)-n-1$, if $X$ is compact, and if $f_{0}$ and $f_{1}$ are isotopic, then they are pth order isotopic.

Proof. Let $\pi_{1}: X \times \tilde{I} \rightarrow X$ be the usual projection onto the first coordinate. Let us consider the vector bundle $\pi_{1}^{-1}\left(T_{p}(X)\right) . \pi_{1}^{-1}\left(T_{p}(X)\right)$ is a vector sub-bundle of $T_{p}(X \times \tilde{I})$. Let $F \in C(X \times \tilde{I}, Y)$. Hence we have $D_{p} T_{p}(F): T_{p}(X \times \tilde{I}) \rightarrow T_{1}(Y)$. It is clear that $D_{p} T_{p}(F)_{(x, t)} \mid \pi_{1}^{-1}\left(T_{p}(X)\right)_{(x, t)}=D_{p} T_{p}\left(f_{t}\right)_{x}$. Hence $F$ is a $p$ th order regular homotopy ( $p$ th order isotopy) between $f_{0}$ and $f_{1}$ if

(1) $f_{t}=f_{0}$ for $t \leqq 0$, and $f_{t}=f_{1}$ for $t \geqq 1$,

(2) $f_{t}$ is itself an immersion (embedding) for each $t$, and

(3) the map $D_{p} T_{p}(F) \mid \pi_{1}^{-1}\left(T_{p}(X)\right)$ is of maximal rank on each fiber. Let us denote $\pi_{1}^{-1}\left(T_{p}(X)\right)$ by $E$. 
We now proceed as in Theorem 6.2, by picking out an admissible submanifold collection $\left(K, K^{\prime}\right)$ of $\operatorname{Hom}\left(E, T_{1}(Y)\right)$, where $K=K_{1} \cup \ldots \cup K_{s}$. The submanifolds $K_{i}$ have the crucial property that if $F \in C(X \times \tilde{I}, Y)$ and if $F$ satisfies (1) and (2) above then $F$ is a $p$ th order regular homotopy ( $p$ th order isotopy) between $f_{0}$ and $f_{1}$ if $D_{E}(F)(X \times \tilde{I}) \cap K_{i}=\varnothing$ for all $i . \operatorname{Hom}\left(E, T_{1}(Y)\right)$ is a vector bundle over $X \times Y$ with projection $\pi$ and fiber $\operatorname{Hom}\left(R^{v(n, p)}, R^{N}\right)$. Let $K_{i}^{\prime}$ be the submanifold of $\operatorname{Hom}\left(R^{v(n, p)}, R^{N}\right)$ consisting of the matrices of rank $i$. Let $U \times V$ be a locally trivializing coordinate neighborhood of $\operatorname{Hom}\left(E, T_{1}(Y)\right)$. We can then define $K_{i}$ by the relation $K_{i} \cap \pi^{-1}(U \times V)=U \times V \times K_{i}^{\prime}$. It is clear that the $K_{i}$ have the desired property.

If $N \geqq v(n, p)$ the $K_{i}$ of minimum codimension is $K_{v(n, p)-1}$, whose codimension is $N-v(n, p)+1$. If $N \leqq v(n, p)$ the $K_{i}$ of minimum codimension is $K_{N-1}$, whose codimension is $v(n, p)-N+1$. Hence $n+1<N-v(n, p)+1$ if and only if $N \geqq v(n, p)+n+1$. Also $n+1<v(n, p)-N+1$ if and only if $N \leqq v(n, p)-n-1$.

Let $A=X \times(-\varepsilon, 0] \cup X \times[1,1+\varepsilon)$. Then $X \times \tilde{I}-A=(0,1) \times X$ is a manifold. Let $F$ be a homotopy between the $f_{0}$ and $f_{1}$ given in the hypotheses to part (a). We may assume that $F$ is smooth. Let

$$
C_{A}(F)=\{G \in C(X \times \tilde{I}, Y)|G| A=F \mid A\} .
$$

Let us assume $N \geqq v(n, p)+n+1$. Hence by 5.4 the set of $G \in C_{A}(F)$ such that $D_{E}(G)(X \times \tilde{I}) \cap K_{i}=\varnothing$, for all $i$, is dense in $C_{A}(F)$. This proves (a) because, in this case condition (2) above is redundant. Let $H=\left\{G \in C(X \times \tilde{I}, Y) \mid g_{t}\right.$ is regular for each $t \in \tilde{I}\}$. It is easy to see that $H$ is open in $C(X \times \tilde{I}, Y)$. Let $H_{A}(F)=H \cap C_{A}(F)$. The hypotheses of (c) say that $H_{A}(F) \neq \varnothing$. If $n<N \leqq v(n, p)-n-1$, the set of $G \in C_{A}(F)$ such that $D_{E}(G)(X \times \tilde{I}) \cap K_{i}=\varnothing$ for all $i$, is dense in $C_{A}(F)$. But $H$ is an open set in $C(X \times \tilde{I}, Y)$ which meets $C_{A}(F)$. Hence there exists a $p$ th order regular homotopy between $f_{0}$ and $f_{1}$. This proves (c). Let $H^{\prime}=\left\{G \in C(X \times \tilde{I}, Y) \mid g_{t}\right.$ is an embedding for each $\left.t\right\}$. It is easy to see that $H^{\prime}$ is an open subset of $C(X \times \tilde{I}, Y)$. $H^{\prime}$ also contains the embeddings of $X \times \tilde{I}$ into $Y$. Hence if $N \geqq 2 n+3, H^{\prime}$ is an open dense subset of $C(X \times \tilde{I}, Y)$. Hence if $N \geqq \max (2 n+3, v(n, p)+n+1)$, the $G^{\prime} \in H^{\prime}$ such that $D_{E}\left(G^{\prime}\right)(X \times \tilde{I}) \cap K_{i}$ $=\varnothing$ for all $i$, is open and dense in $C(X \times \tilde{I}, Y)$. Therefore by the exact same method we used to prove 5.2, we could show that the $G \in C_{A}(F)$ such that $D_{E}(G)(X \times \tilde{I}) \cap K_{i}=\varnothing$ for all $i$ and such that $G \in H^{\prime}$ are dense in $C_{A}(F)$. Hence we have shown part (b). We prove part (d) by combining the arguments used to prove part (c) and part (b) in the obvious manner.

COROLlaRY 7.3. Let $X$ be a smooth manifold of dimension $n$. Let $Y=R^{N}$ with an arbitrary set of dissections $\left(D^{(k)}\right), k=1,2, \cdots$, associated with it. Then if $N \geqq v(n, p)+n+1$, any two pth order nondegenerate immersions of $X$ into $R^{N}$ are pth order regularly homotopic. Furthermore if 


$$
N \geqq \max (v(n, p)+n+1,2 n+3)
$$

any two pth order nondegenerate embeddings of $X$ in $\boldsymbol{R}^{N}$ are pth order isotopic provided $X$ is compact.

REMARK. When $p=1$ the first statement of 7.3 implies the following.

Corollary 7.4. Any two immersions of an n-manifold $X$ into $R^{2 n+1}$ are regularly homotopic.

COROllary 7.5. Let $X$ be an n-manifold. Let $Y=R^{N}$ with the set of dissections $\left(D^{(k)}\right), k=1,2, \cdots$. If $2 n+1 \leqq N \leqq v(n, p)-n-1$, then any two pth order nondegenerate immersions of $X$ into $R^{N}$ are pth order regularly homotopic.

REMARK. If $X=S^{1}$, the circle, the problem of classifying the $p$ th order regular homotopy classes of $p$ th order nondegenerate immersions of $S^{1}$ into $R^{N}$ is an open question only for $N=p-1, p$, and $p+1$. The rest of the cases are taken care of by 7.5 and 7.3 , because $v(n, p)=p$.

8. $T_{p}(X)$ and its characteristic classes. In this section we will glean what bundle theoretic information we can about $T_{p}(X)$ from the preceding sections. We will then define some characteristic classes for these and related bundles.

Let $n<N$. Let $G_{n, N}$ and $V_{n, N}$ stand for the Grassmann manifold of $n$-planes in $N$-space, and the Stiefel manifold of $n$-frames in $N$-space, respectively. $V_{n, N}$ is a principal $O(n)$ bundle over $G_{n, N}$. Let $\xi_{n, N}$ be the associated vector bundle of fiber dimension $n$ over $G_{n, N}$.

Let $E \rightarrow{ }^{\pi} X$ and $F \rightarrow^{\pi^{\prime}} Y$ be two smooth vector bundles over smooth manifolds $X$ and $Y$. Let $f: E \rightarrow F$ be a vector bundle homomorphism covering $\tilde{f}: X \rightarrow Y$.

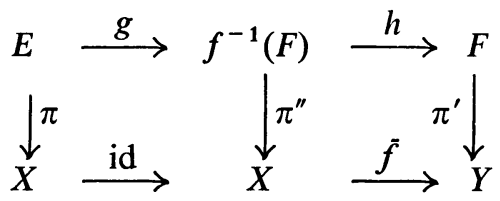

Let $\pi^{\prime \prime}: \tilde{f}^{-1}(F) \rightarrow X$ be the pullback of $F$ over $X$. Then as is well known (Milnor [1]) we can factor $f$ into $h g$, where $h$ is a vector bundle homomorphism from $\bar{f}^{-1}(F)$, into $F$, over $f$, which is an isomorphism on each fiber. $g$ is a vector bundle homomorphism over $X$ such that $g\left|E_{x}=f\right| E_{x}$, for each $x \in X$. Whenever we are in this situation we will represent $g$ by $f$ !

Finally if $\xi$ is a vector bundle over $X$ let $W(\xi)$ stand for the total StiefelWhitney class, and $W_{i}(\xi)$ for the $i$ th Stiefel-Whitney class of $\xi$.

Definition 8.1. Let $X$ and $Y$ be smooth manifolds of dimension $n$ and $N$, respectively. Let $\left(D^{(k)}\right), k=1,2, \cdots$, be a sequence of $k$ th order dissections on $Y$. Let $f: X \rightarrow Y$ be a $p$ th order nondegenerate immersion of $X$ in $Y$. 
(a) Let $N \geqq v(n, p) . D_{p} T_{p}(f)$ !: $T_{p}(X) \rightarrow f^{-1}(T(Y))$ is a monomorphism on each fiber and hence a monomorphism of vector bundles over $X$. Let $N_{f, Y}^{p}(X)$ $=$ cokernel $\left(D_{p} T_{p}(f) !\right) . N_{f, Y}^{p}(X)$ is called the pth order normal bundle of $X$ in $Y$ with respect to $f$. If $Y=R^{N}$, we denote $N_{f, Y}^{p}(X)$ by $N_{f}^{p}(X)$ and just call it the pth order normal bundle of $X$ with respect to $f$.

(b) Let $N \leqq v(n, p)$. $D_{p} T_{p}(f)$ !: $T_{p}(X) \rightarrow f^{-1}(T(Y))$ is an epimorphism of vector bundles over $X$. Let $K_{f, Y}^{p}(X)=\operatorname{Kernel}\left(D_{p} T_{p}(f) !\right) . K_{f, Y}^{p}(X)$ is called the pth order kernel bundle of $X$ in $Y$ with respect to $f$ (pth order co-normal bundle of $X$ in $Y$ with respect to $f)$. Similarly if $Y=R^{N}, K_{f, Y}^{p}(X)$ is denoted by $K_{f}^{p}(X)$ and is called the pth order kernel bundle of $X$ with respect to $f$ (pth order co-normal bundle of $X$ with respect to $f$ ).

Proposition 8.2. Let $X$ and $Y$ be manifolds of dimension $n$ and $N$, respectively. Let $\left(D^{(k)}\right), k=1,2, \cdots$, be a sequence of kth order dissections on $Y$. Let $f: X \rightarrow Y$ be pth order nondegenerate immersion of $X$ in $Y$.

(a) Let $N \geqq v(n, p)$. Then $N_{f, Y}^{p}(X)$ is a vector bundle of fiber dimension $N-v(n, p)$. Furthermore, we have the following short exact sequence of vector bundles over $X$.

$$
0 \longrightarrow T_{p}(X) \stackrel{D_{p} T_{p}(f) !}{\longrightarrow} f^{-1}(T(Y)) \longrightarrow N_{f, X}^{p}(X) \longrightarrow 0 .
$$

(b) Let $N \leqq v(n, p)$. Then $K_{f, Y}^{p}(X)$ is a vector bundle of fiber dimension $v(n, p)-N$. Moreover we have the following short exact sequence of vector bundles over $X$.

$$
0 \longrightarrow K_{f, Y}^{p}(X) \longrightarrow T_{p}(X) \stackrel{D_{p} T_{p}(f) !}{\longrightarrow} f^{-1}(T(Y)) \longrightarrow 0 .
$$

Proof. This is an immediate consequence of Definition 8.1.

COROLlary 8.3. (a) Under the hypotheses of 8.2(a), we have

$$
f^{-1}(T(Y)) \cong T_{p}(X) \oplus N_{f, Y}^{p}(X)
$$

(b) Under the hypotheses of 8.2(b), we have

$$
T_{p}(X) \cong f^{-1}(T(Y)) \oplus K_{f, Y}^{p}(X) .
$$

Proof. This follows immediately from 8.2 and the fact that every short sequence of smooth vector bundles over a smooth paracompact manifold has a smooth splitting.

Proposition 8.4. Let $X$ be a smooth n-manifold. Let $Y=R^{N}$, with an arbitrary set of kth order dissection on it.

(a) If $N>v(n, p)$, the existence of a pth order nondegenerate immersion of $X$ in $R^{N}$ implies the existence of a vector bundle $\xi$ of fiber dimension $N-v(n, p)$ such that

$$
T_{p}(X) \oplus \xi=X \times R^{N}
$$


(b) If $N<v(n, p)$, the existence of a pth order nondegenerate immersion $X$ in $R^{N}$ implies the existence of a bundle $\alpha$ of fiber dimension $v(n, p)-N$ such that, $X \times R^{N} \oplus \alpha \cong T_{p}(X)$, and $T_{1}(X)$ is contained in the trivial bundle.

Proof. (a) Let $f: X \rightarrow R^{N}$ be the $p$ th order nondegenerate immersion. Let $\xi=N_{f}^{p}(X)$, and recall the fact that the pull back of a trivial bundle is trivial. Hence (a) follows from 8.3(a).

(b) Let $f: X \rightarrow R^{N}$ be the $p$ th order nondegenerate immersion. Let $\eta=f^{-1}\left(T_{1}(Y)\right)$. $\eta \supseteq T_{1}(X)$ because $f$ is an immersion. Let $\alpha=K_{f}^{p}(X)$. The proposition follows from $8.3(\mathrm{~b}) .6 .2$ and 6.4 yield the following corollary.

Corollary 8.5. Assume the hypotheses of 8.4.

(a) There exists a vector bundle $\xi$ of fiber dimension $n$ such that, $\xi \oplus T_{p}(X) \cong X \times R^{N}$.

(b) There exists a vector bundle $\alpha$ of fiber dimension $n$ such that for $p>1$ and $n>1$,

$$
\alpha \oplus\left(X \times R^{v(n, p)-n}\right) \cong T_{p}(X)
$$

REMARK. 8.5(b) shows that in some sense $T_{p}(X)$ is not just a trivial bundle added onto $T_{1}(X)$, in that $\alpha$ which is the nontrivial part of $T_{p}(X)$ is complementary to $T_{1}(X)$.

Before going on to its characteristic classes, we will give a geometric description of the classifying map of $T_{p}(X)$.

Definition 8.6. Let $X$ be an $n$-manifold. Let $\left(D^{(k)}\right), k=1,2, \cdots$, be a sequence of $k$ th order dissections on $R^{N}$. Let $\langle$,$\rangle be the usual inner product on R^{N}$, and let $P$ be the exponential map associated with that Riemann structure. Assume that $N>v(n, p)$. Let $f: X \rightarrow R^{N}$ be a $p$ th order nondegenerate immersion. This defines a smooth map $G_{T}^{p}(f): X \rightarrow G_{v(n, p), N}$ and $G_{N}^{p}(f): X \rightarrow G_{N-v(n, p), N}$ as follows. Let $x \in X . G_{T}^{p}(f)(x)$ is equal to the $v(n, p)$-plane through the origin of $R^{N}$, parallel to the $v(n, p)$-plane $P D_{p} T_{p}(f)_{x}\left(T_{p}(X)_{x}\right)$, through $f(x) . G_{N}^{p}(f)(x)$ is the $N-v(n, p)$ plane which is the orthogonal complement to the $v(n, p)$-plane $G_{T}^{p}(f)(x) . G_{T}^{p}(f)$ and $G_{N}^{p}(f)$ are called the pth order Gauss tangential map associated to $f$, and the pth order Gauss normal map associated to $f$, respectively.

Under the hypotheses of 8.6 it is clear that $T_{p}(X)=G_{T}^{p}(f)^{-1}\left(\xi_{v(n, p), N}\right)$ and $N_{f}^{p}(X)=G_{N}^{p}(f)^{-1}\left(\xi_{N-v(n, p), N}\right)$. Now $V_{v(n, p), v(n, p)+n+1}$ is $n$-connected, therefore $G_{v(n, p), v(n, p)+n+1}$ is the universal classifying space for vector bundles of fiber dimension $v(n, p)$ over $X$ with $\xi_{v(n, p), v(n, p)+n+1}$ the universal vector bundle. Hence if $f: X \rightarrow R^{v(n, p)+n+1}$ is a $p$ th order nondegenerate immersion with respect to the chosen dissections on $R^{v(n, p)+n+1}$ then $G_{T}^{p}(f)$ is the classifying map for $T_{p}(X)$. Similarly $G_{N}^{p}(f): X \rightarrow G_{n+1, v(n, p)+n+1}$ is the classifying map of the $p$ th order normal bundle $N_{f}^{p}(X)$. The classifying theorem for bundles tells us that if 
$f^{\prime}: X \rightarrow R^{v(n, p)+n+1}$ is another $p$ th order nondegenerate immersion, then $G_{T}^{p}\left(f^{\prime}\right) \cong G_{T}^{p}(f)$. However, we know by 7.3 that there exists a $p$ th order regular homotopy $f_{t}: X \rightarrow R^{v(n, p)+n+1}$ from $f$ to $f^{\prime}$. Hence the homotopy between $G_{T}^{p}(f)$ and $G_{T}^{p}\left(f^{\prime}\right)$ can be given by $G_{T}^{p}\left(f_{t}\right)$. Furthermore $G_{N}^{p}\left(f_{t}\right)$ establishes a homotopy between $G_{N}^{p}(f)$ and $G_{N}^{p}\left(f^{\prime}\right)$. Hence $N_{f}^{p}(X)$ and $N_{f^{\prime}}^{p}(X)$ are isomorphic. Therefore, we have shown the following proposition.

Proposition 8.7. Let $X$ be a smooth n-manifold. Let $\left(D^{(k)}\right)$ be any sequence of kth order dissections on $R^{v(n, p)+n+1}$. Let $f_{0}$ and $f_{1}$ be two given pth order nondegenerate immersions from $X$ into $R^{v(n, p)+n+1}$.

(1) Then $G_{T}^{p}\left(f_{i}\right), i=0,1$, are classifying maps for $T_{p}(X)$, and the homotopy between them has the form $G^{p}\left(f_{t}\right)$ where $f_{t}$ is a pth order regular homotopy between $f_{0}$ and $f_{1}$.

(2) If $p>1$, the maps $G_{N}^{p}\left(f_{i}\right), i=0,1$, are classifying maps for the $N_{f_{i}}^{p}(X)$ $i=0,1$, respectively. The pth order regular homotopy $\left(f_{t}\right)$ in (1) induces a homotopy $G_{N}^{p}\left(f_{t}\right)$ between the $G_{N}^{p}\left(f_{i}\right)$. Hence $N_{f_{0}}^{p}(X) \cong N_{f_{1}}^{p}(X)$.

DEFinition 8.8. Let $W^{p}(X)=W\left(T_{p}(X)\right)$, and set $\overline{W^{p}(X)}=W^{p}(X)^{-1} \cdot W^{p}(X)$ is called the total pth order Stiefel-Whitney class of $X$, and $\overline{W^{p}(X)}$ is called the total dual pth order Stiefel-Whitney class of $X . W_{i}^{p}(X)$ and $\overline{W_{i}^{p}(X)} \in H^{i}\left(X, Z_{2}\right)$ are called the ith, pth order S.-W. class of $X$ and the ith dual pth order S.-W. class of $X$, respectively.

Remark. Let $f: X \rightarrow R^{N}$ be a $p$ th order nondegenerate immersion of $X$ into $R^{N}$, with respect to arbitrary dissections on $R^{N}$. Then if $N \geqq v(n, p)$, Whitney duality immediately implies that $\overline{W^{p}(X)}=W\left(N_{f}^{p}(X)\right)$.

We will now make a short digression into algebraic topology in order to state a theorem that will aid our calculating $W^{p}(X)$. Let $\xi$ be a vector bundle of fiber dimension $n$. Then let $W(\xi)=1+W_{1}(\xi)+W_{2}(\xi)+\cdots+W_{n}(\xi)$ be the total Stiefel-Whitney class of $\xi$. We then perform the following formal factorization.

$$
1+W_{1}(\xi) x+W_{2}(\xi) x^{2}+\cdots+W_{n}(\xi) x^{n}=\prod_{i=1}^{n}\left(1+\sigma_{i} x\right) .
$$

We then know that $W_{k}(\xi)$ is the $k$ th elementary symmetric function in the $\sigma_{i}$ 's. With this construction we have the following fact, the proof of which is an easy exercise in the use of techniques found in E. Thomas [1].

LEMMA 8.9. $W\left(0^{p} \xi\right)=\prod_{1 \leqq i_{1} \leqq \ldots \leqq i_{p} \leqq n}\left(1+\left(\sigma_{i_{1}}+\cdots+\sigma_{i_{p}}\right) x\right)$.

We know that the bundles $\left(T_{k}(X)\right)$ satisfy the short exact sequences of vector bundles

$$
0 \longrightarrow T_{k-1}(X) \longrightarrow T_{k}(X) \longrightarrow 0^{k} T(X) \longrightarrow 0 \text {. }
$$


Hence by Whitney duality, $W^{k}(X)=W^{k-1}(X) \cup W\left(0^{k} T(X)\right)$. This coupled with the fundamental theorem on symmetric polynomials gives us the following result.

Proposition 8.10. $W_{i}^{p}(X)$ is a polynomial in the classes $W_{j}(X), j \leqq i$. The explicit formula for the polynomial can be computed from the formula

$$
W^{p}(X)=W(X) \cup W\left(0^{2} T(X)\right) \cup \cdots \cup W\left(0^{p} T(X)\right) .
$$

COROLlary 8.11. $T_{p}(X)$ is orientable if $T_{1}(X)$ is orientable.

Finally, Definition 8.8 coupled with Proposition 8.4 yields the following result.

Proposition 8.12. Let $X$ be a smooth n-manifold. Let $\left(D^{(k)}\right)$ be an arbitrary sequence of $k$ th order dissections on $R^{N}:$ Let $1 \leqq s<n$.

(a) If $X$ is pth order nondegenerately immersible into $R^{v(n, p)+n-s}$, then $\overline{W_{s+1}^{p}(X)}=\cdots=\overline{W_{n}(X)}=0$.

(b) If $X$ is pth order nondegenerately immersible into $R^{v(n, p)-n+s}$, then $W_{s+1}^{p}(X)=\cdots=W_{n}^{p}(X)=0$.

9. The $p$ th order normal form of an immersion. Let $X$ and $Y$ be smooth manifolds of dimension $n$ and $N$, respectively. Let $\left(D^{(k)}\right), k=1,2, \cdots$, be a sequence of $k$ th order dissections on $Y$. We know that, for fixed $k, D^{(k)}$ is a splitting of the short exact sequence of vector bundles

$$
0 \longrightarrow T_{k}(Y) \underset{D^{(k)}}{\stackrel{i_{k}}{\rightleftarrows--}} T_{k+1}(Y) \stackrel{P_{k}}{\longrightarrow} 0^{k+1} T_{1}(Y) \longrightarrow 0 .
$$

Let $f: X \rightarrow Y$ be a smooth map. As before we set $D_{P} T_{p}(f)=D^{(1)} \cdots D^{(p-1)} T_{P}(f)$.

PROPOSITION 9.1. $D_{P} T_{p}(f) \mid T_{p-k}(X)=D_{p-k} T_{p-k}(f)$.

Proof.

$$
\begin{aligned}
D_{P} T_{p}(f)\left(i_{p-1} \cdots i_{p-k}\right) & =D^{(1)} \cdots D^{(p-1)} i_{p-1} \cdots i_{p-k} T_{p-k}(f) \\
& =D^{(1)} \cdots D^{(p-k-1)} T_{p-k}(f) \\
& =D_{p-k} T_{p-k}(f) .
\end{aligned}
$$

Definition 9.2. Let $v(n, p)$ be the fiber dimension of $T_{p}(X)$. Let us assume that $N \geqq v(n, p)$ and that $f$ is a $p$ th order nondegenerate immersion. This implies that $f$ is a $k$ th order nondegenerate immersion for all $k \leqq p$. Let $N_{f, Y}^{p}(X)$ be the $p$ th order normal bundle of $X$ in $Y$ with respect to $f$. Let us consider the following diagram of vector bundles over $X$, with the bottom row and left-hand column 


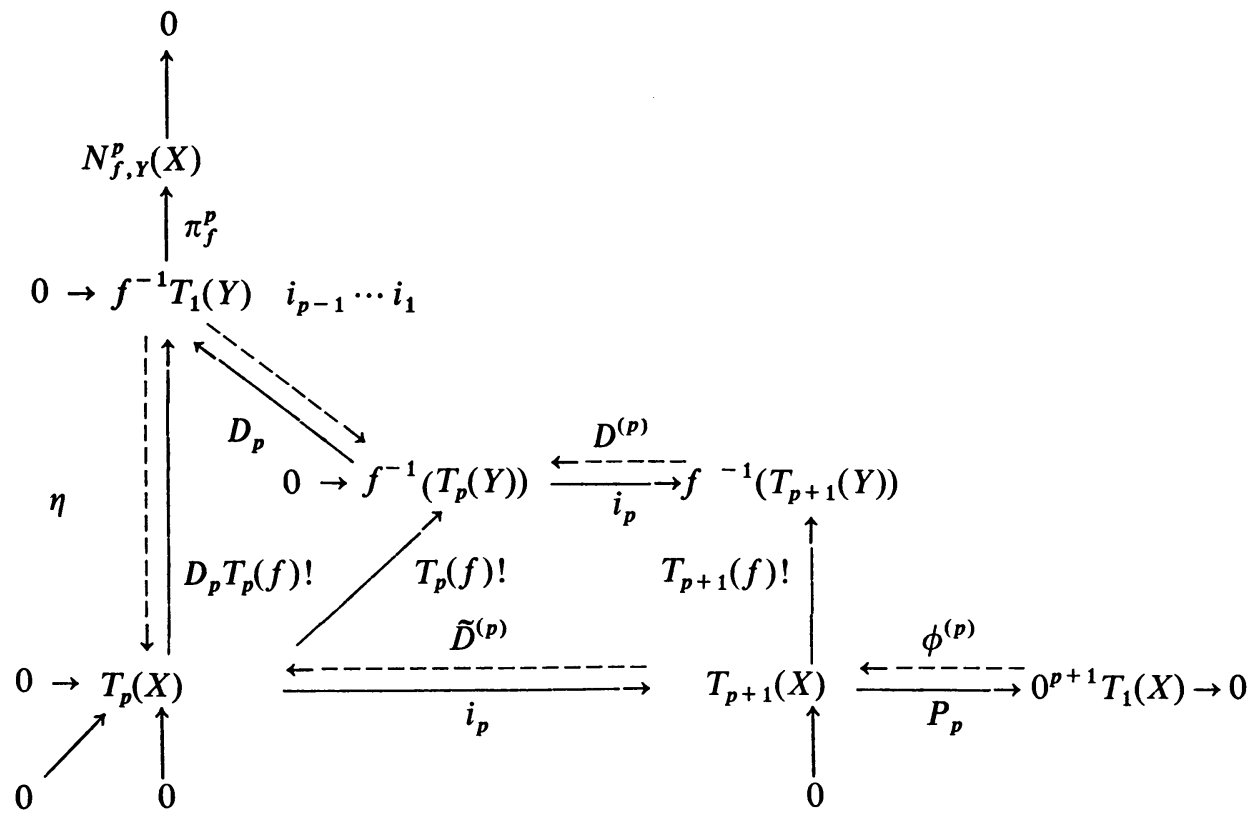

DIAGRAM 1

exact. By the vector bundle homomorphisms over $X, i_{p}$ and $D^{(k)}$ between the $f^{-1} T_{k}(Y)$ will be meant the pull-backs of the usual vector bundle homomorphisms over $Y, i_{p}$ and $D^{(k)}$ between the $T_{k}(Y)$.

Let us look at $\pi_{f}^{p} D_{p+1} T_{p+1}(f) !: T_{p+1}(X) \rightarrow N_{f, Y}^{p}(X)$.

$$
\pi_{f}^{p} D_{p+1} T_{p+1}(f) ! i_{p}=\pi_{f}^{p} D_{p} T_{p}(f) !=0 .
$$

Hence $\pi_{f}^{p} D_{p+1} T_{p+1}(f)$ ! vanishes on the image of $i_{p}$. Therefore this induces a unique vector bundle homomorphism over $X v_{f}^{p}: 0^{p+1} T(X) \rightarrow N_{f, Y}^{p}(X)$. The map $v_{f}^{p}$ is called the pth normal form of the pth order nondegenerate immersion $f$.

Proposition 9.3. Let $\eta: f^{-1} T(Y) \rightarrow T_{p}(X)$ be a splitting of the vertical short exact sequence of Diagram 1.

(a) Let $\xi=\eta D^{(1)} \ldots D^{(p-1)}$. Then $\xi T_{p}(f) !=\operatorname{id}_{T_{p}(X)}$.

(b) Let $\tilde{D}^{(p)}=\xi D^{(p)} T_{p+1}(f)$ !. Then $\tilde{D}^{(p)}$ is a splitting of the horizontal short exact sequence of Diagram 1 . Hence $\tilde{D}^{(p)}$ yields a pth order dissection on $X$.

(c) Let $\phi^{(p)}: 0^{p+1} T_{1}(X) \rightarrow T_{p+1}(x)$ be the splitting "on the right" of the horizontal short exact sequence of Diagram 1, induced by $\tilde{D}^{(p)}$ : Then $v_{f}^{p}=\pi_{f}^{p} D_{p+1} T_{p+1}(f) ! \phi^{(p)}$. 
Proof.

(a)

$$
\begin{aligned}
\xi T_{p}(f) ! & =\eta D^{(1)} \ldots D^{(p-1)} T_{p}(f) ! \\
& =\eta D_{p} T_{p}(f) ! \\
& =\operatorname{id}_{T_{p}(X)} . \\
\tilde{D}^{(p)} i_{p} & =\xi D^{(p)} T_{p+1}(f) ! i_{p} \\
& =\xi D^{(p)} i_{p} T_{p}(f) ! \\
& =\xi T_{p}(f) ! \\
& =\operatorname{id}_{T_{p}(X)} .
\end{aligned}
$$

(c) Let $x \in X$ and let $u_{1}, \cdots, u_{p+1} \in T(X)_{x}$. We defined $v_{f}^{p}\left(u_{1} \cdot \cdots \cdot u_{p+1}\right)$ by noting that it does not make any difference on which element of $P_{p}^{-1}\left(u_{1} \cdots \cdot u_{p+1}\right)$ we evaluate $\pi_{f}^{p} D_{p+1} T_{p+1}(f)$ !. However we know that $P_{p} \phi^{(p)}=\mathrm{id}_{0^{p+1} T(X)}$. Hence $\phi^{(p)}\left(u_{1} \cdot \cdots \cdot u_{p+1}\right) \in P_{p}^{-1}\left(u_{1} \cdots \cdot u_{p+1}\right)$.

REMARK. Let $p=1$, and let $D^{1}$ be the dissection induced by a Riemann connection on $Y$. Let $\eta$ be the splitting induced by the inner product in $f^{-1} T_{1}(Y)$. Then the first normal form $v_{f}^{1}: T_{1}(X) \circ T_{1}(X) \rightarrow f^{-1} T_{1}(Y)$ is in some classical works called the second fundamental form of the immersion $f$. It is this fact that will enable us to apply this machinery to some classical problems in "Riemannian singularities." This will form the major part of the successor to this paper.

We will adopt the following convention. Let $E$ and $F$ be smooth vector bundles over $X$ of fiber dimension $n$ and $p$, respectively. Let $q=\min (n, p)$. Let $h \in S(\operatorname{Hom}(E, F) \mid X)$. Then $S_{a}(h)=\{x \in X \mid \operatorname{rank}(h(x))=q-a\}$.

Proposition 9.4. $S_{a}\left(D_{p+1} T_{p+1}(f) !\right)=S_{a}\left(v_{f}^{p}\right)$.

Proof.

$$
S_{a}\left(v_{f}^{p}\right)=\left\{x \in X \mid \operatorname{dim}\left(\operatorname{ker}\left(v_{f}^{p}(x)\right)\right)=a\right\}
$$

or

$$
S_{a}\left(v_{f}^{p}\right)=\left\{x \in X \mid \operatorname{dim}\left(\operatorname{ker}\left(v_{f}^{p}(x)\right)\right)=a+v(n, p+1)-N\right\}
$$

as $N \geqq v(n, p+1)$ or $N<v(n, p+1)$, respectively. Similarly

$$
S_{a}\left(D_{p+1} T_{p+1}(f) !\right)=\left\{x \in X \mid \operatorname{dim}\left(\operatorname{ker}\left(D_{p+1} T_{p+1}(f) !(x)\right)\right)=a\right\}
$$

or

$$
S_{a}\left(D_{p+1} T_{p+1}(f) !\right)=\left\{x \in X \mid \operatorname{dim}\left(\operatorname{ker}\left(D_{p+1} T_{p+1}(f) !(x)\right)\right)=a+v(n, p+1)-N\right\}
$$

as $N \geqq v(n, p+1)$ or $N<v(n, p+1)$, respectively. Hence it will suffice to show that $\operatorname{dim}\left(\operatorname{ker}\left(v_{f}^{p}(x)\right)\right)=\operatorname{dim}\left(\operatorname{ker}\left(D_{p+1} T_{p+1}(f) !(x)\right)\right)$ for every $x \in X$. This will follow from the following lemma.

LeMMA 9.5. Let $\phi: V_{1} \rightarrow V_{2}$ be a linear map between two finite dimensional vector spaces. Let $A_{1}$ and $A_{2}$ be vector subspaces of $V_{1}$ and $V_{2}$ respectively. 
Assume that $\phi\left(A_{1}\right) \subset A_{2}$ and that $\phi$ maps $A_{1}$ isomorphically onto $A_{2}$. Let $\tilde{\phi}: V_{1} / A_{1} \rightarrow V_{2} / A_{2}$ be the induced map. Then $\operatorname{dim}(\operatorname{ker}(\phi))=\operatorname{dim}(\operatorname{ker}(\tilde{\phi}))$.

Proof. $\operatorname{dim}\left(V_{1}\right)=\operatorname{dim}(\operatorname{ker}(\phi))+\operatorname{dim}(\operatorname{im}(\phi))$, and $\operatorname{dim}\left(V_{1} / A_{1}\right)$ $=\operatorname{dim}(\operatorname{ker}(\tilde{\phi}))+\operatorname{dim}(\operatorname{im}(\tilde{\phi}))$.

$$
\operatorname{dim}\left(V_{1}\right)-\operatorname{dim}(\operatorname{im}(\phi))=\operatorname{dim}\left(V_{1} / A_{1}\right)-\operatorname{dim}(\operatorname{im}(\tilde{\phi}))
$$

because $\phi$ is an isomorphism from $A_{1}$ onto $A_{2}$. Hence $\operatorname{dim}(\operatorname{ker}(\phi))=\operatorname{dim}(\operatorname{ker}(\tilde{\phi}))$.

9.4 follows from 9.5 if we set $A_{2}=D_{p} T_{p}(f) !\left(T_{p}(X)_{x}\right), A_{1}=T_{p}(X)_{x}, V_{1}=T_{p+1}(X)_{x}$, $V_{2}=f^{-1} T_{1}(Y)_{x}$ and $\phi=D_{p+1} T_{p+1}(f) !(x)$.

Corollary 9.6. $D_{p+1} T_{p+1}(f)$ ! is injective (surjective) if and only if $v_{f}^{p}$ is injective (surjective) as a homomorphism of vector bundles over $X$.

Proposition 9.7. Let $k \leqq p$. Then we have the following short exact sequence of vector bundles over $X$.

$$
0 \longrightarrow v_{f}^{k-1}\left(0^{k} T_{1}(X)\right) \longrightarrow N_{f, Y}^{k-1}(X) \longrightarrow N_{f, Y}^{k}(X) \longrightarrow 0 .
$$

Proof. Consider the following commutative diagram of vector bundles with exact rows and columns.

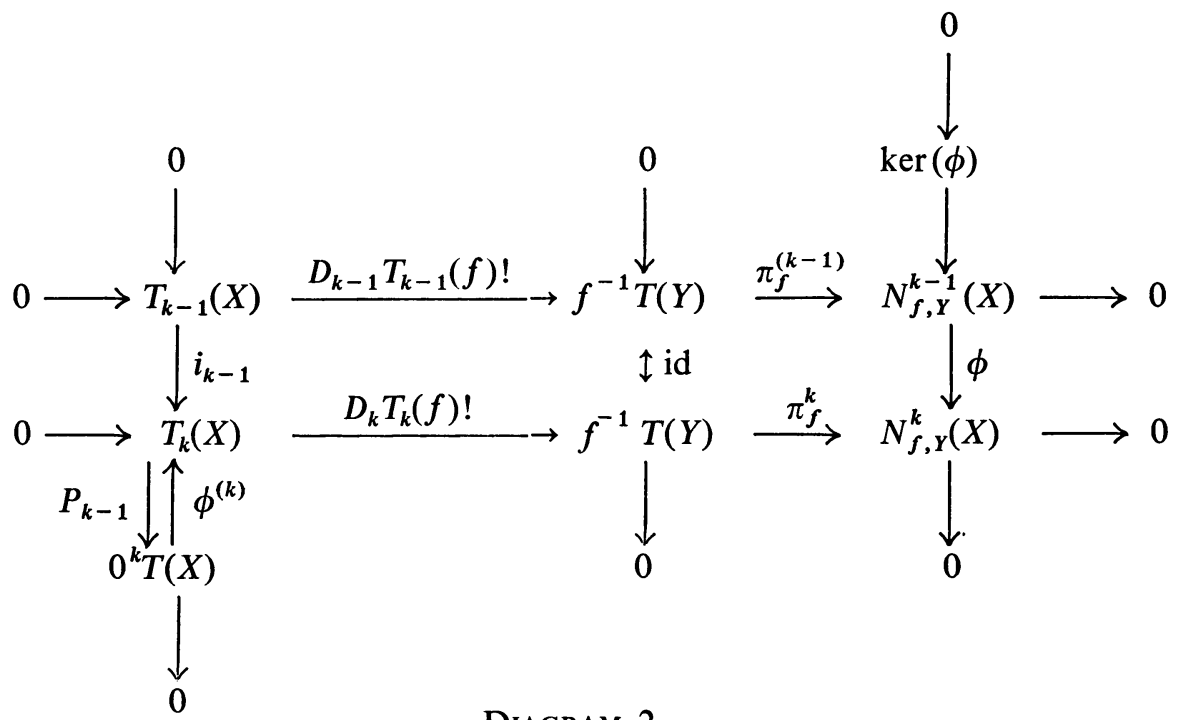

\section{Diagram 2}

Let $\phi$ be the vector bundle homomorphism from $N_{f, Y}^{k-1}(X)$ to $N_{f, Y}^{k}(X)$ induced from $i_{k}$, the identity, and the fact that the left-hand square of Diagram 2 commutes. $\phi$ is clearly an epimorphism. The "Noetherian" isomorphism theorem tells us that $\operatorname{ker} \phi \cong 0^{k} T(X)$. It remains to show that the isomorphism is given by $v_{f}^{k-1}$. Let $\phi^{(k)}$ be a splitting of the left-hand vertical exact sequence of Diagram 2. Then $v_{f}^{k-1}=\pi_{f}^{k-1} D_{k} T_{k}(f) ! \phi^{(k)}$. Then 


$$
\begin{aligned}
\phi v_{f}^{k-1} & =\phi \pi_{f}^{k-1} D_{k} T_{k}(f) ! \phi^{(k)} \\
& =\pi_{f}^{k} D_{k} T_{k}(f) ! \phi^{(k)} \\
& =0 .
\end{aligned}
$$

Hence $v_{f}^{k-1}\left(0^{k} T(X)\right) \subseteq \operatorname{ker} \phi$, and therefore $v_{f}^{k-1}\left(0^{k} T_{1}(X)\right)=\operatorname{ker} \phi$.

COROLlary 9.8. If $k \leqq p$,

$$
N_{f, Y}^{1}(X) \cong v_{f}^{1}\left(0^{2} T(X)\right) \oplus \cdots \oplus v^{k-1}\left(0^{k} T(X)\right) \oplus N_{f, Y}^{k}(X) .
$$

The above gives us some insight into the behavior of nondegenerate immersions.

10. Counterexamples. In this section we will calculate $W^{p}(X)$ and $\bar{W}^{p}(X)$ for some manifolds $X$, with the aim of showing that in general the theorems of $\$ 6$ are optimal. We know that every manifold $X$ of dimension $\geqq 2$ can be $p$ th order nondegenerately immersed in $R^{v(n, p)+n}$, and if $p>1$, in $R^{v(n, p)-n}$. Hence by 8.12 if $W_{n}^{p}(X) \neq 0$, or if $W_{n}^{p}(X) \neq 0$, then $X$ can not be $p$ th order nondegenerately immersed in $R^{v(n, p)+n-1}$, or then $X$ can not be $p$ th order nondegenerately immersed in $R^{v(n, p)-n+1}$, respectively.

We will now calculate $W\left(0^{p} T_{1}(X)\right)$ for manifolds $X$ of dimension 2 . By the construction near the end of $\S 8$ we have that

$$
W\left(0^{p} T_{1}(X)\right)=\prod_{1 \leqq i_{1} \leqq \ldots \leqq i p \leqq 2}\left[1+\left(\sigma_{i_{1}}+\cdots+\sigma_{i_{p}}\right) x\right] .
$$

Hence if we assume that $p=2 k$, then

$$
W\left(0^{p} T_{1}(X)\right)=\left(1+W_{1}(X)\right)^{k}=1+k W_{1}(X)+\frac{k(k-1)}{2} W_{1}(X)^{2} .
$$

When $p=2 k+1$ we have

Hence

$$
\begin{aligned}
W\left(0^{p} T_{1}(X)\right) & =\left(1+W_{1}(X)+W_{1}(X)^{2}\right)^{k+1} \\
& =1+(k+1) W_{1}(X)+(k+1)\left(\frac{k+2}{2}\right) W_{1}(X)^{2} .
\end{aligned}
$$

$$
W\left(0^{p} T_{1}(X)\right)= \begin{cases}1 & \text { if } p=8 s \\ 1+W_{1}(X)+W_{1}(X)^{2} & \text { if } p=8 s+1 \\ 1+W_{1}(X) & \text { if } p=8 s+2 \\ 1+W_{1}(X)^{2} & \text { if } p=8 s+3 \\ 1+W_{1}(X)^{2} & \text { if } p=8 s+4 \\ 1+W_{1}(X) & \text { if } p=8 s+5 \\ 1+W_{1}(X)+W_{1}(X)^{2} & \text { if } p=8 s+6 \\ 1 & \text { if } p=8 s+7, \text { for } s=0,1,2, \cdots .\end{cases}
$$


We will now calculate $W\left(0^{2} T_{1}(X)\right)$, where the dimension of $X$ is 3 .

$$
\begin{aligned}
W\left(0^{2} T(X)\right) & =\left(1+\left(\sigma_{1}+\sigma_{2}\right) x\right)\left(1+\left(\sigma_{1}+\sigma_{3}\right) x\right)\left(1+\left(\sigma_{2}+\sigma_{3}\right) x\right) \\
& \left.=\left(1+\left(\sigma_{2}+\sigma_{3}\right) x+\left(\sigma_{1}^{2}+\sigma_{2} \sigma_{3}+\sigma_{1} \sigma_{3}+\sigma_{1} \sigma_{2}\right) x^{2}\right)\right)\left(1+\left(\sigma_{2}+\sigma_{3}\right) x\right) \\
& =\left(1+\left(\sigma_{1}^{2}+\sigma_{1} \sigma_{2}\right) x^{2}+\left(\sigma_{1}^{2} \sigma_{2}\right) x^{3}+\text { the remainder mod } 2\right) \\
& =1+\left(W_{1}(X)^{2}+W_{2}(X)\right) x^{2}+\left(W_{1}(X) W_{2}(X)+W_{3}(X)\right) x^{3} .
\end{aligned}
$$

Finally we will calculate $W\left(0^{2} T(X)\right)$, where the dimension of $X$ is 4 . $W\left(0^{2} T(X)\right)=$

$$
\begin{aligned}
&\left(1+\left(\sigma_{1}+\sigma_{2}\right) x\right)\left(1+\left(\sigma_{1}+\sigma_{3}\right) x\right)\left(1+\left(\sigma_{1}+\sigma_{4}\right) x\right)\left(1+\left(\sigma_{2}+\sigma_{3}\right) x\right) \\
& \cdot\left(1+\left(\sigma_{2}+\sigma_{4}\right) x\right)\left(1+\left(\sigma_{3}+\sigma_{4}\right) x\right) .
\end{aligned}
$$

We now expand and collect the terms containing $\sigma_{1}$. Hence we have $W\left(0^{p} T(X)\right)=$

$$
\begin{array}{r}
\left(1+\sigma_{1} x+\sigma_{1}^{2} x^{2}+\left(\sigma_{1}^{3}+\sigma_{1}^{2} \sigma_{2}+\sigma_{1}^{2} \sigma_{3}+\sigma_{1} \sigma_{2} \sigma_{3}+\sigma_{1}^{2} \sigma_{4}+\sigma_{1} \sigma_{2} \sigma_{4}+\sigma_{1} \sigma_{3} \sigma_{4}\right) x^{3}+\cdots\right) \\
\left.\cdot\left(1+\left(\sigma_{3}+\sigma_{4}\right)\right) x+\left(\sigma_{2}^{2}+\sigma_{3} \sigma_{4}+\sigma_{2} \sigma_{4}+\sigma_{2} \sigma_{3}\right) x^{2}\right)\left(1+\left(\sigma_{3}+\sigma_{4}\right) x\right)
\end{array}
$$

Let us count the various monomials containing $\sigma_{1}$. Then apply the formulas in Milnor [1] and we get

$$
\left.W\left(0^{2} T(X)\right)=1+W_{1}(X)+W_{1}(X)^{2}+W_{1}(X)^{3}+\left(W_{2}(X)\right)^{2}+W_{1}(X) W_{3}(X)\right)
$$

Proposition 10.1. Let $X$ be a smooth manifold.

(a) If the dimension of $X$ is 2 , then

$$
W^{p}(X)= \begin{cases}1 & \text { when } p=8 s \\ 1+W_{1}(X)+W_{1}(X)^{2} & \text { when } p=8 s+1 \\ 1 & \text { when } p=8 s+2 \\ 1+W_{1}(X)^{2} & \text { when } p=8 s+3 \\ 1 & \text { when } p=8 s+4 \\ 1+W_{1}(X) & \text { when } p=8 s+5 \\ 1 & \text { when } p=8 s+6 \\ 1 & \text { when } p=8 s+7, \text { for } s=0,1,2, \cdots .\end{cases}
$$

(b) If the dimension of $X$ is 3 , then

$$
W^{2}(X)=1+W_{1}(X)+W_{1}(X)^{2}+W_{1}(X)^{3} .
$$


(c) If the dimension of $X$ is 4 , then

$$
W^{2}(X)=1+W_{2}(X)+\left(W_{1}(X) W_{2}(X)+W_{3}(X)\right) .
$$

Proof. (a) and (b) are immediate consequences of 8.10 and the foregoing calculations. (c) requires the additional fact that

$$
W_{4}(X)=W_{1}(X)^{4}+W_{1}(X)^{2} W_{2}(X)+W_{2}(X)^{2}+W_{4}(X)=0
$$

for a 4-manifold.

Corollary 10.2. Let $X$ be a smooth manifold.

(a) Let $X$ be a 2-manifold. Then

$$
W^{p}(X)= \begin{cases}1 & \text { if } p=8 s, 8 s+2,8 s+4,8 s+6, \text { and } 8 s+7 \\ 1+W_{1}(X) & \text { if } p=8 s+1 \\ 1+W_{1}(X)^{2} & \text { if } p=8 s+3 \\ 1+W_{1}(X)+W_{1}(X)^{2} & \text { if } p=8 s+r, \text { for } s=0,1,2, \cdots .\end{cases}
$$

(b) Let $X$ be a 3-manifold. Then $\bar{W}^{2}(X)=1+W_{1}(X)$.

(c) Let $X$ be a 4-manifold. Then

$$
W^{2}(X)=1+W_{2}(X)+\left(W_{1}(X) W_{2}(X)+W_{3}(X)\right)+W_{2}(X)^{2} .
$$

THEOREM 10.3. Let $R^{N}$ have an arbitrary sequence of $k$ th order dissections on it.

(a) $P_{2}(R)$ can not be pth order nondegenerately immersed in $R^{v(2, p)+1}$, if $p=8 s+3$ or if $p=8 s+5$, for $s$ any non-negative integer.

(b) $P_{2}(C)$ can not be second order nondegenerately immersed in $R^{v(2,4)+3}$.

(c) $P_{2}(R)$ can not be pth order nondegenerately immersed in $R^{v(n, p)-1}$, if $p=8 s+1$ or if $p=8 s+3$, for $s$ any non-negative integer.

Proof. If $X=P_{2}(R)$, then $H^{*}\left(P_{2}(R), Z_{2}\right)=Z_{2}[\alpha] / \alpha^{3}$, the truncated polynomial ring generated by $\alpha \in H^{\prime}\left(P_{2}(R) ; Z_{2}\right)$. Furthermore $W\left(P_{2}(R)\right)=1+\alpha+\alpha^{2}$. Hence $\alpha^{2}=W_{1}(X)^{2} \neq 0$. Therefore $10.3(\mathrm{a})$ and $10.3(\mathrm{c})$ are implied by $10.1,10.2$ and 8.12. We similarly know that $H^{*}\left(P_{2}(C), Z_{2}\right)=Z_{2}[\alpha] / \alpha^{3}, \alpha \in H^{2}\left(P_{2}(C), Z_{2}\right)$. It is furthermore well known that $W_{2}\left(P_{2}(C)\right)=\alpha$. Hence $W_{2}\left(P_{2}(C)\right)^{2} \neq 0$. Hence 8.12 and 10.2 imply $10.3(b)$.

REMARK. In 10.3(a) and in 10.3(b), a nondegenerate immersion of the required order exists by the theorems of $\S 6$, if we allow ourselves one more dimension in the target space. A similar statement is true about 10.3(c).

It is clear that with some more calculations one could construct many more examples. Whether 6.2 is optimal, with $Y$ Euclidean space for each pair $(n, p)$, is an open question. 


\section{APPENDIX ON THE FUNCTION SPACE TOPOLOGIES}

This appendix contains the definitions and proofs of some of the basic facts concerning the topologies one can put on the function spaces of smooth maps between manifolds. These facts are apparently less well know than they should be. This discussion is based in part on the notes of $M$. Hirsch (see Hirsch [1, pp. 15-22]). In particular the proof of Proposition A.8 is taken from Hirsch's notes. Finally all the facts used on the topology of inverse limits can be found in the appropriate section of Eilenberg-Steenrod.

Let $X$ and $Y$ be smooth manifolds. Let $C^{p}(X, Y)$ denote the set of $p$ times differentiable continuous functions from $X$ to $Y$. For each $f \in C^{p}(X, Y)$ we have the map $T_{p}(f): T_{p}(X) \rightarrow T_{p}(Y)$. This induces a map $e_{p}: C^{p}(X, Y) \rightarrow$ $C^{0}\left(X, \operatorname{Hom}\left(T_{p}(X), T_{p}(Y)\right)\right)$ defined by $e_{p}(f)_{x}=T_{p}(f)_{x}$, for $f \in C^{p}(X, Y)$ and $x \in X$. $e_{p}$ is clearly one-one and hence we can consider $C^{p}(X, Y)$ as a subset of $C^{0}\left(X, \operatorname{Hom}\left(T_{p}(X), T_{p}(Y)\right)\right)$.

Definition A.1. Let $\tau^{p}$ be the topology on $C^{p}(X, Y)$ induced from the compact open topology on $C^{0}\left(X, \operatorname{Hom}\left(T_{p}(X), T_{p}(Y)\right)\right) . \tau^{p}$ is called the $C^{p}$-topology of compact convergence.

If $p \leqq q$ then $C^{q}(X, Y) \subset C^{p}(X, Y)$. If we topologize $C^{q}(X, Y)$ with $\tau^{q}$ and $C^{p}(X, Y)$ with $\tau^{p}$ the inclusion map $i_{p}^{q}$ is continuous. Thence the set of spaces $\left(C^{p}(X, Y)\right.$ with topology $\left.\tau^{p}\right)$ and maps $i_{p}^{q}$ form an inverse mapping system. Let $C(X, Y)=\operatorname{inv} \lim C^{p}(X, Y)$. This set is nothing other than the $C^{\infty}$ functions from $X$ to $Y$.

Definition A.2. Let $\tau$ be the inverse limit topology on $C(X, Y)$. The pair $(C(X, Y), \tau)$ is called the $C^{\infty}$ functions with the $C^{\infty}$ topology of compact convergence.

Hence we know that $C(X, Y)$ with this topology is a Hausdorff space, because the $\left(C^{p}(X, Y), \tau^{p}\right)$ are Hausdorff spaces.

We now give another description of this topology which will motivate its name. We know that $\operatorname{Hom}\left(T_{p}(X), T_{p}(Y)\right)$ is a smooth manifold satisfying the second axiom of countability. Hence it is well known that $\operatorname{Hom}\left(T_{p}(X), T_{p}(Y)\right)$ admits a Riemann metric, such that the associated metric space topology given on the manifold by the arc-length function $D_{p}$, is complete. Let $\left(\phi_{i}, U_{i}\right), i=1,2, \cdots$, be an admissible atlas on $X$. Pick $f$ and $g$ in $C^{p}(X, Y)$. Let

$$
\rho_{i ; p}(f, g)=\max _{x \in \bar{B}_{i}}\left(D_{p}\left(e_{p}(f)_{x}, e_{p}(g)_{x}\right)\right)
$$

Let $\bar{\tau}_{p}$ be the uniform topology on $C^{p}(X, Y)$ determined by the countable family of pseudo-metrics $\left\{\rho_{i ; p}\right\}$. Intuitively this topology should be the one called the $C^{p}$-topology of compact convergence, as it is the topology induced on $C^{p}(X, Y)$ from the topology of uniform convergence on compacta on 


$$
C_{0}\left(X, \operatorname{Hom}\left(T_{p}(X), T_{p}(Y)\right)\right) \text {. }
$$

However as is well known (Kelley $[1$, p. 230]), the topology of compact convergence and the compact open topology are equivalent. Hence $\bar{\tau}^{p}=\bar{\tau}^{p}$. $\tau^{p}$ is given by a countable family of pseudo-metrics. Hence $\left(C^{p}(X, Y), \bar{\tau}^{p}\right)$ is a metric space with the metric $\rho_{p}=\sum_{i=1}^{\infty} 2^{-i} \rho_{i, p} /\left(I+p_{i}, p\right)$. Furthermore an easy local argument will show it is a complete metric space. Let $\rho_{i}=\sum_{p=0}^{\infty} \rho_{i, p} /\left(1+\rho_{i, p}\right)$, where the $\left\{\rho_{i, p}\right\}$ are constructed as above for all $p \geqq 0$. Let $\bar{\tau}$ be the topology in $C(X, Y)$ induced by the pseudo-metrics $\left\{\rho_{i}\right\}$. In that the inverse limit topology is taken from the product topology, we have that the pair $(C(X, Y), \tau)$ is the inverse limit of the pairs $\left(C^{p}(X, Y), \bar{\tau}^{p}\right)$ with the usual inclusions. Hence the topology $\bar{\tau}$ is equivalent to $\tau$. Now by the same argument as above the metric

$$
\rho=\sum_{i=1}^{\infty} \frac{1}{2^{i}} \frac{\rho_{i}}{1+\rho_{i}}
$$

turns $(C(X, Y), \bar{\tau})$ into a metric space. It is furthermore a complete metric space because the $C^{p}(X, Y)$ were and each $C^{p}(X, Y)$ is a Hausdorff space. Hence $C(X, Y)$ is a closed subset in the inverse limit topology of $\prod_{p \geqq 0} C^{p}(X, Y)$ with the product topology. But a closed subset of a complete space is complete. Hence $C(X, Y)$ is also a complete metric space.

Definition A.3. A topological space $X$ is called a Baire space if the intersection of a countable family of open dense subsets of $X$ is itself dense in $X$.

It is well known that complete metric spaces are Baire spaces. Hence we have shown:

Proposition A.4. The spaces $C^{p}(X, Y)$ and $C(X, Y)$ with the $C^{p}$ topology of compact convergence and the $C^{\infty}$ topology of compact convergence, respectively, are Baire spaces.

We will now construct the second set of topologies we are interested in. Let $D_{p}$ be a metric on $\operatorname{Hom}\left(T_{p}(X), T_{p}(Y)\right)$, compatible with the manifold topology. Let $\delta$ be a positive continuous function on $X$. Let $f \in C^{p}(X, Y)$. Set $S_{\delta}^{p}(f)=\left\{g \in C^{p}(X, Y) \mid D_{p}\left(e_{p}(f)_{x}, e_{p}(g)_{x}\right)<\delta(x)\right\}$. Let $\sigma^{p}$ be the topology on $C^{p}(X, Y)$ obtained by taking the sets $S_{\rho}^{p}(f)$ one for each positive continuous function $\delta$ on $X$, as the neighborhood system of $f \in C^{p}(X, Y)$. It is easy to see that this topology is independent of the metric $D_{p}$ chosen. As before the sets $\left(C^{p}(X, Y)\right.$ with the topology $\sigma^{p}$ ) together with the inclusion maps form an inverse mapping system. Hence let the pair $(C(X, Y), \sigma)$ be the $C^{\infty}$-functions, with the inverse limit topology induced from the $\sigma^{p}$. It is clear that both $\sigma^{p}$ and $\sigma$ are Hausdorff topologies.

We now offer another description of $\sigma$ and $\sigma^{p}$. Let $\left(\phi_{i}, U_{i}\right), i=1,2, \cdots$, be an admissible atlas on $X$. Let $D_{p}$ be the metric of the above paragraph. If $f, g \in C^{p}(X, Y)$, let $\left\{\rho_{i, p}\right\}$ be the pseudo-metrics defined by $\rho_{i, p}(f, g)=\max _{x \in \bar{B}_{i}}\left(D_{p}\left(e_{p}(f)_{x}, e_{p}(g)_{x}\right)\right)$. 
Let $\left\{\varepsilon_{i}\right\}, i=1,2, \cdots$, be a sequence of positive real numbers. Then $S_{\left\{\varepsilon_{i}\right\}}^{p}(f)$ $=\left\{g \in C^{p}(X, Y) \mid \rho_{i, p}(f, g)<\varepsilon_{i}\right.$ for every $\left.i\right\}$. Let $\bar{\sigma}^{p}$ be the topology defined by letting the sets $S_{\left\{\varepsilon_{i}\right\}}^{p}(f)$, one for each sequence $\left\{\varepsilon_{i}\right\}, i=1,2, \cdots$, be the neighborhood system of $f$.

Proposition A.5. The topology $\sigma^{p}$ is equivalent to the topology $\bar{\sigma}^{p}$.

Proof. Let $f \in C^{p}(X, Y)$. Let $\delta$ be a positive continuous function on $X$. Let $\varepsilon_{i}=\inf _{x \in \bar{B}_{i}} \delta(x) . \varepsilon_{i}>0$ because $\bar{B}_{i}$ is compact. Hence $S_{\left(\varepsilon_{i}\right)}^{p}(f) \subset S_{\delta}^{p}(f)$.

Conversely let $\left(\varepsilon_{i}\right)$ be a family of positive numbers. We are finished if we can construct a continuous function $\delta>0$ on $X$ such that $\delta(x)<\varepsilon_{i}$ on each $\bar{B}_{i}$. Each $x \in X$ has a neighborhood $W_{x}$ which meets at most a finite number of the $\bar{B}_{i}$; say $\bar{B}_{i_{1}}, \cdots, \bar{B}_{i_{s}}$. Let $\varepsilon_{w_{x}}$ be a positive real number such that

$$
0<\varepsilon_{w}<\inf \left(\varepsilon_{i_{1}}, \cdots, \varepsilon_{i_{s}}\right) .
$$

These neighborhoods $\left(W_{x}\right)$ form an open cover of $X$. Pick a locally finite refinement $\left(V_{j}\right)_{j \in J}$ of the $\left(W_{x}\right)$. Let $\left(\phi_{j}\right)_{j \in J}$ be a smooth partition of unity subordinate to the cover $\left\{V_{j}\right\}_{j \in J}$. For each $j \in J$ pick a $W_{x}^{\prime}$ such that $V_{j} \subset W_{x}^{\prime}$. Set $\varepsilon_{j}^{\prime}=\varepsilon_{w}$. We now define

$$
\delta(x)=\sum_{j \in J} \phi_{j}(x) \varepsilon_{j}^{\prime}
$$

It is easy to show that this function has the desired properties.

Corollary A.6. Let $\bar{\sigma}$ be the inverse limit topology on $C(X, Y)$ induced by the $\bar{\sigma}^{p}$. Then $\sigma$ and $\bar{\sigma}$ are equivalent topologies on $C(X, Y)$.

As before set $\rho_{i}=\Sigma_{p \geqq 0} 2^{-p} \rho_{i, p} /\left(1+\rho_{i, p}\right)$. Let $\left(\varepsilon_{i}\right), i=1,2, \cdots$, be a sequence of positive real numbers. Let us set $S_{\left(\varepsilon_{i}\right)}(f)=\left\{g \in C(X, Y) \mid \rho_{i}(f, g)<\varepsilon_{i}\right.$ for every $\left.i\right\}$. If we take the sets $S_{\left(\varepsilon_{i}\right)}(f)$ one for each family $\left(\varepsilon_{i}\right)$, as a fundamental system of neighborhoods of $f$, the topology induced on $C(X, Y)$ is clearly equivalent to $\sigma$. Hence we have the following proposition.

Proposition A.7. Let $\sigma$ and $\tau$ be the two topologies defined above for $C(X, Y)$. Then there exist bases $B_{\sigma}$ and $B_{\tau}$ of $\sigma$ and $\tau$ respectively such that each element of $B_{\sigma}$ is the intersection of a countable family of $\tau$-closures of elements of $B_{\tau}$. Furthermore $\sigma$ is finer than $\tau$.

Proposition A.8. The space $C(X, Y)$ with the topology $\sigma$ is a Baire space.

Proof. Let $\left(A_{n}\right), n=1,2, \cdots$, be a sequence of $\sigma$-open and $\sigma$-dense subsets of $C(X, Y)$. Let $B_{\sigma}$ and $B_{\tau}$ be bases for $\sigma$ and $\tau$ respectively satisfying the conditions of Proposition A.7. In that $\tau$ is weaker than $\sigma$, each $A_{n}$ is $\tau$-dense. We know $A_{n}$ can be expressed as $\bigcap_{k=1}^{\infty} A_{n, k}$ with each $A_{n, k} \tau$-open. Since $A_{n} \subset A_{n, k}$, each $A_{n, k}$ is $\tau$-dense. Let $U \in B_{\sigma} . A_{n} \cap U$ is dense in the topology induced on $U$ by $\sigma$, by as- 
sumption. Hence $A_{n} \cap U$ is also $\tau$-dense in the $\tau$ topology on $U$. We know by Proposition A. 7 that $U$ is $\tau$-closed. Hence $U$ is a complete metric space and therefore a Baire space. Hence

$$
\bigcap_{n, k} A_{n, k} \cap U \neq \varnothing \text { implies } \bigcap_{n} A_{n} \cap U \neq \varnothing .
$$

From now on whenever we talk about the space of functions $C(X, Y)$ we will mean this set with the topology $\sigma$. My reason for picking this topology, which appears to be the less natural of the two, is that the immersions and embeddings are open subsets of $C(X, Y)$, even when $X$ is not compact. Finally, it is easy to show that the topologies $\sigma$ and $\tau$ are equivalent if and only if $X$ is compact.

\section{BIBLIOGRA PHY}

\section{R. Abraham}

1. Transversality in manifolds of mappings, Bull. Amer. Math Soc. 69 (1963), 470-474.

W. Ambrose, R. Palais and I. Singer

1. Sprays, An. Acad. Brasil. Ci. 32 (1960), 163-178.

M. F. AtiYah

1. Complex analytic connections in fibre bundles, Trans. Amer. Math. Soc. 85 (1957), 181-206.

J. ErLLS

1. Singularities of smooth maps, mimeographed notes, Columbia University, New York, 1959.

S. EILENBERg AND N. STEENRod

1. Foundations of algebraic topology, Princeton Mathematical Series No. 15, Princeton Univ. Press, Princeton, N. J., 1952.

E. A. Feldman

1. The geometry of immersions, Bull. Amer. Math. Soc. 69 (1963), 693-698.

R. GODEMENT

1. Topologie algébrique et théorie des faisceaux, Actualités Sci. Indust. No. 1252, Hermann, Paris, 1958.

M. HIRSCH

1. Differential topology, mimeographed notes, Univ. of California, Berkeley, Calif., 1962.

J. KELLEY

1. General topology, Van Nostrand, New York, 1955.

S. KOBAYASHI

1. Frame bundles of higher order contact, Proc. Sympos. Pure Math. Vol. 3, Differential Geometry, pp. 196-193, Amer. Math. Soc., Providence, R. I., 1961.

J.-L. KoszuL

1. Lectures on fibre bundles and differential geometry, Tata Institute of Fundamental Research, Bombay, 1960.

H. LEVINE

1. Singularities of differentiable mappings, mimeographed notes, Bonn University, Bonn, 1959.

P. LIBERMANN

1. Calcul tensoriel et connexions d'ordre superieur, 3è Coloquio Brasileiro de Matematica, Fortaleza, 1961.

J. MiLNOR

1. Characteristic classes, mimeographed notes, Princeton University, Princeton, N. J., 1957.

2. Differential topology, mimeographed notes, Princeton University, Princeton, N. J., 1959. 
J. MunKRes

1. Elementary differential topology, Annals of Mathematics Studies No. 54, Princeton Univ. Press, Princeton, N. J., 1963.

W. F. PoHL

1. Differential geometry of higher order, Topology 1 (1962), 169-211.

2. Connexions in differential geometry of higher order, Applied Mathematics and Statistics Laboratories, Stanford University, Stanford, Calif., 1963.

A. SARD

1. Images of critical sets, Ann. of Math. 68 (1958), 247-259.

J.-P. SERRE

1. Faisceaux algébriques cohérents, Ann. of Matb. 61 (1955), 197-278.

N. STEENROD

1. The topology of fibre bundles, Princeton Mathematical Series No. 14, Princeton Univ. Press, Princeton, N. J., 1951.

D. J. STRuiK

1. Differential geometry, Addison-Wesley, Reading, Mass., 1950.

E. THomas

1. On tensor products of n-plane bundles, Arch. Math. Karlsruhe 10 (1959), 174-179.

H. WHITNEY

1. Differentiable manifolds, Ann. of Math. 37 (1936), 645-680.

2. On singularities of mappings of Euclidean spaces. I. Mappings of the plane into the plane, Ann. of Math. 62 (1955), 374-410.

3. Geometric integration theory, Princeton Mathematical Series No. 21, Princeton Univ. Press, Princeton, N. J., 1957.

4. Elementary structure of real algebraic varieties, Ann. of Math. 66 (1957), 545-556.

5. On regular closed curves in the plane, Compositio Math. 4 (1937), 276-284.

6. The singularities of $n$-manifold in (2n-1)-space, Ann. of Math. 45 (1944), 247-293.

Princeton University, Princeton, New Jersey 